\title{
Spectral shape of the UV ionizing background and He II absorption at redshifts $1.8<z<2.9^{\star}$
}

\author{
I. I. Agafonova ${ }^{1, \star \star}$, S. A. Levshakov ${ }^{1, \star \star}$, D. Reimers ${ }^{1}$, C. Fechner $^{1}$, D. Tytler ${ }^{2}$, R. A. Simcoe ${ }^{3}$, and A. Songaila ${ }^{4}$ \\ 1 Hamburger Sternwarte, Universität Hamburg, Gojenbergsweg 112, 21029 Hamburg, Germany \\ e-mail: lev@astro.ioffe.rssi.ru \\ 2 Department of Physics and Center for Astrophysics and Space Sciences, University of California, San Diego, MS 0424, La Jolla, \\ CA 92093-0424, USA \\ 3 MIT Center for Space Research, 77 Massachusetts Ave, 37-664B, Cambridge, MA 02139, USA \\ ${ }^{4}$ Institute of Astronomy, University of Hawaii, 2680 Woodlawn Drive, Honolulu, HI 96822, USA
}

Received 30 May 2006 / Accepted 10 October 2006

\section{ABSTRACT}

\begin{abstract}
Aims. The shape of the UV ionizing background is reconstructed from optically thin metal absorption-line systems identified in spectra of HE 2347-4342, Q 1157+3143, and HS 1700+6416 in the redshift interval $1.8<z<2.9$.

Methods. The systems are analyzed by means of the Monte Carlo Inversion method completed with the spectral shape recovering procedure.

Results. The $U V B$ spectral shape fluctuates at $2.4<z<2.9$ mostly due to radiative transfer processes in the clumpy IGM. At $z \lesssim 1.8$, the IGM becomes almost transparent both in the H I and He II Lyman continua and the variability of the spectral shape comes from diversity of spectral indices describing the QSO/AGN intrinsic radiation. At $z>2.4$, the recovered spectral shapes show intensity depression between 3 and 4 Ryd due to He II Ly $\alpha$ absorption in the IGM clouds (line blanketing) and continuous medium (true Gunn-Petersen effect). The mean He II Ly $\alpha$ opacity estimated from the depth of this depression corresponds within 1-2 $\sigma$ to the values directly measured from the $\mathrm{HI} / \mathrm{He}$ II Ly $\alpha$ forest towards the quasars studied. The observed scatter in $\eta=N(\mathrm{He}$ II $) / N(\mathrm{HI})$ and anti-correlation between $N(\mathrm{HI})$ and $\eta$ can be explained by the combined action of variable spectral softness and differences in the mean gas density between the absorbing clouds. Neither of the recovered spectral shapes show features which can be attributed to the putative input of radiation from soft sources like starburst galaxies.
\end{abstract}

Key words. cosmology: observations - line: formation - line: profiles - quasars: absorption lines quasars: general - cosmology: diffuse radiation

\section{Introduction}

This paper continues our study of the UV ionizing background $(U V B)$ of the metagalactic radiation in the range 1-10 Ryd as reconstructed from optically thin QSO metal absorption systems. In the previous papers, our analysis of the absorbers at $z \approx 3$ (Agafonova et al. 2005, hereafter Paper I) and in the range $1.46<z<1.8$ (Reimers et al. 2006, hereafter Paper II) has led to the following conclusions:

1. At $z \approx 3$, the spectral shape of the UV ionizing radiation shows a sharp reduction in flux in the energy range 3 Ryd $<E<4$ Ryd which can be interpreted as manifestation of the He II Gunn-Petersen effect - He II Ly $\alpha$ continuous absorption in the intergalactic medium.

2. At $z<1.8$, the ionizing spectra turn out to be much harder at $E \geq 3$ Ryd as compared to model spectra of Haardt \& Madau (1996, hereafter HM). This indicates that intergalactic clouds with $N(\mathrm{H} \mathrm{I})>10^{15} \mathrm{~cm}^{-2}$ responsible for the He II break (absorption in the He II Lyman continuum) become more rare at low $z$ than was supposed by the cloud statistics in HM.

^ Based on observations obtained at the VLT Kueyen telescope (ESO), the W. M. Keck Observatory and the HST.

$\star \star$ On leave from Ioffe Physico-Technical Institute, St. Petersburg, Russia.
3. The ionizing background both at $z \approx 3$ and at $z<1.8$ is dominated by radiation from QSO/AGNs. The input of the soft radiation from galaxies/stars is negligible, limiting the escape fraction of the galactic UV photons to $f_{\text {esc }}<0.05$.

In the present paper, we reconstruct the underlying $U V B$ using metal absorbers from the range $1.8<z<2.94$ identified in spectra of three bright QSOs: HE 2347-4342 $\left(z_{\mathrm{em}}=\right.$ 2.88), Q $1157+3143\left(z_{\mathrm{em}}=2.96\right)$, and HS $1700+6416\left(z_{\mathrm{em}}=\right.$ 2.74). In the considered redshift range, the shape of the $U V B$ is closely related to the process of He II reionization in course of cosmic time. Recent observations of the $\mathrm{HI} / \mathrm{He}$ II Ly $\alpha$ forest towards HE 2347-4342 (Shull et al. 2004; Zheng et al. 2004, hereafter Z04) and HS 1700+6416 (Fechner et al. 2006a, hereafter F06) revealed (i) significant fluctuations of the ratio $\eta=N(\mathrm{He}$ II $) / N(\mathrm{HI}),($ ii $)$ anti-correlation between $\eta$ and $N(\mathrm{HI})$, and (iii) decrease of median $\eta$ towards lower $z$. Although a large part of the scatter of $\eta$ seems to be artificial and caused by noise in He II data, errors in H I and He II column densities, unidentified blends etc. (see F06), similarity of the results obtained both for the HE 2347-4342 and HS 1700+6416 sightlines supposes that spatial fluctuations of $\eta$ do really exist. These fluctuations suggest a variable softness $S=J_{912} / J_{228}$ of the metagalactic radiation field with higher $\eta$ value corresponding to softer $U V B$. A diversity of the spectral indices describing QSO continuum radiation, an input of radiation from soft sources of type starburst 
galaxies, and radiative transfer through clumpy media are the reasons usually considered for the $U V B$ variability. Calculations of the $U V B$ spectra accounting for all these effects are still well beyond modern computational facilities, although some attempts to explain the observed scatter of $\eta$ via the modeling of the $\mathrm{HI} / \mathrm{He}$ II Ly $\alpha$ forest have been made (Maselli \& Ferrara 2005; Bolton et al. 2006). Thus, direct reconstructions of the $U V B$ spectral shape from metal absorption-line systems at different redshifts and along different sightlines can help to clarify the character of the $U V B$ fluctuations and to determine the sources of the ionizing background.

The paper is organized as follows. Section 2 contains some qualitative information outlining main steps in the $U V B$ recovering procedure. Selected absorption systems are analyzed in Sect. 3. The obtained results are discussed and summarized in Sects. 4 and 5.

\section{Recovering the shape of the underlying radiation field}

Computational procedure used to recover the spectral shape of the ionizing radiation from optically thin metal systems is described in Paper I. Here we briefly outline the basics needed to understand the results presented below in Sect. 3 .

Absorption systems are analyzed by means of the Monte Carlo Inversion (MCI) procedure (Levshakov et al. 2000, hereafter LAK; Levshakov et al. 2002, 2003a,b). The MCI is based on the assumption that all lines observed in the absorption system are formed in a cloud where the gas density and velocity are random functions of the coordinate $x$ along the cross line. It is also assumed that the metal abundance throughout the cloud is constant, the gas is in thermal and ionization equilibrium and optically thin for the ionizing UV radiation. This implies that ion fractions are determined entirely by the gas density $n_{\mathrm{H}}(x)$ (or, equivalently, by the ionization parameter $\left.U(x) \propto 1 / n_{\mathrm{H}}\right)$ and by the spectral energy distribution of the background ionizing radiation in the range 1-10 Ryd (defined by the ionization edges of the observed metal ions - from Si II to O VI, see Fig. 1).

Since the gas density varies from point to point along the sightline, the ion fractions vary as well. Thus, for a given point within the line profile the observed intensity results from a mixture (superposition) of different ionization states of the gas due to irregular Doppler shifts of the local absorption coefficient (see Fig. 1 in LAK). The dependence of the ion fraction on the gas density (ionization curve) is different for each ion and this is why the line profiles differ in spite of being produced by the same gas cloud.

The following physical parameters are directly estimated in the computational procedure: the mean ionization parameter $U_{0}$, the total hydrogen column density $N_{\mathrm{H}}$, the line-of-sight velocity dispersion, $\sigma_{\mathrm{v}}$, and density dispersion, $\sigma_{\mathrm{y}}$, of the bulk material $\left[y \equiv n_{\mathrm{H}}(x) / n_{0}\right]$, and the chemical abundances $Z_{\mathrm{a}}$ of all elements included in the analysis. With these parameters we can further calculate the column densities for different species $N_{\mathrm{a}}$, and the mean kinetic temperature $T_{\text {kin }}$. Given the absolute intensity of the UV background, the mean gas number density, $n_{0}$, and the line-of-sight thickness, $L$, of the absorber can be evaluated as well.

An advanced version of the MCI completed with the procedure of the spectral shape recovering (referred to as MCISS hereafter) is iterative and works as follows. At first, some basic ionizing spectrum (e.g. HM for corresponding redshift) is taken as an initial approximation. To calculate the ionization curves needed

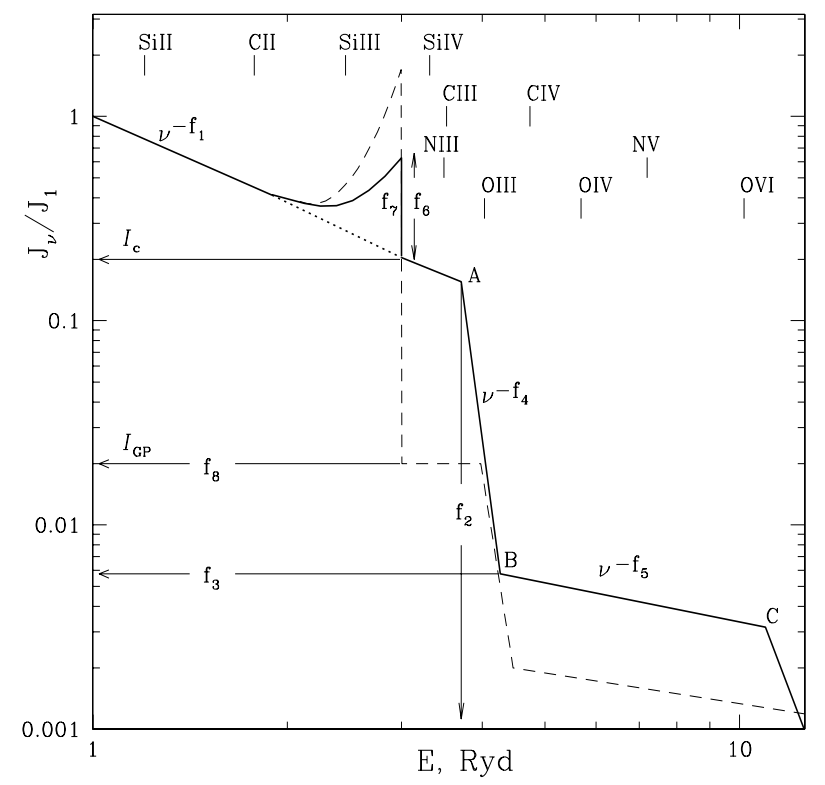

Fig. 1. Schematic picture of metagalactic ionizing spectrum (solid thick line) as predicted by model calculations (Haardt \& Madau 1996; Fardal et al. 1998). The spectrum is normalized so that $J_{v}(h v=1 \mathrm{Ryd})=1$. The emission bump at 3 Ryd is caused by reemission of He II Ly $\alpha$, He II two-photon continuum emission and He II Balmer continuum emission from intergalactic clouds. The positions of ionization thresholds of different ions are indicated by tick marks. The definition of factors $\left\{f_{i}\right\}$ is given in Sect. 2. Dashed line shows the spectral shape recovered from metal absorbers at $z \sim 3$. The continuum depression between 3 and 4 Ryd $\left(I_{\mathrm{c}}-I_{\mathrm{GP}}\right)$ is due to He II Ly $\alpha$ absorption in the IGM (the He II Gunn-Peterson effect).

to carry out the MCI calculations this basic $U V B$ is inserted into the photoionization code CLOUDY (Ferland 1997) together with some guesses about relative element abundances and metallicity. Then the MCI run is performed with the ionization curves taken as external parameters. If the resulting (recovered) relative abundances and the gas metallicity differ from the initial guess, the ionization curves are re-calculated with these current quantities and new MCI runs are performed till the recovered metallicity and element ratios correspond to those used to calculate the ionization curves. If this solution reproduces all line profiles within their uncertainties and no other peculiarities arise (like relative overabundances of elements which are well beyond observational and theoretical constraints), then we can consider the assumed ionizing spectrum as consistent with observations. If, however, the line intensities come over-/underestimated and/or relative element ratios are odd, then the spectral shape of the ionizing radiation is to be adjusted.

The spectral shape adjustment procedure itself is developed on base of the response surface methodology used in the theory of experimental design. It includes $(i)$ the parameterization of the spectral shape by means of a set of variables (called "factors"), (ii) the choice of a quantitative measure (called "response") to evaluate goodness of a trial spectral shape, and (iii) the estimation of a direction in the factor space which leads to a spectrum with the better goodness. Moving along this direction, we come from the initial spectral shape to the one with better characteristics concerning the fitting of the observed line intensities. Now the MCI calculations are carried out with this newly obtained ionizing spectrum and the whole procedure is repeated till the optimal spectral shape is found, i.e. one which allows to 
reproduce the observed intensities of all lines without any physical inconsistencies.

How well the spectral shape can be recovered depends on the number of metal lines involved in the analysis: the more lines of different ionic transitions of different elements are detected in the absorption system, the better constrained is the allowable set of the spectral shapes. Any available a priori information concerning, e.g., the expected element ratios should be also taken into consideration in order to distinguish between possible solutions.

Figure 1 schematically shows the spectrum of the metagalactic radiation as obtained in model calculations (Haardt \& Madau 1996; Fardal et al. 1998) and illustrates its parameterization by a set of factors. These are: $f_{1}-$ the slope (power law exponent) between 1 Ryd and the He II ionization break; $f_{2}$ - the energy at which the He II break starts (point $A$ ); $f_{3}$ - the depth of the He II ionization break, $\log \left(J_{\mathrm{B}} / J_{\mathrm{A}}\right) ; f_{4}-$ the slope between $A$ and $B ; f_{5}-$ the slope between $B$ and $C ; f_{6}, f_{7}-$ the height and width of the He II re-emission bump. The energy of the far UV cut off (point $C$ ) when taken above 100 Ryd does not effect the fractional ionizations of ions we are interesting in. In all computations described in the subsequent sections this energy and the slope after it are kept fixed at $128 \mathrm{Ryd}$ and -1.5 , respectively.

Studying the $U V B$ at $z \approx 3$, we found that metagalactic ionizing spectra reveal a sharp depression in the intensity between 3 and 4 Ryd which was interpreted as manifestation of the He II Gunn-Petersen (GP) effect ${ }^{1}$ (Paper I). Thus, additional factor $f_{8}$ was introduced to describe the depth of the GP depression approximated by a straight step. Knowing this depth, $I_{\mathrm{GP}}$, and the continuum level $I_{\mathrm{c}}$ at $E=3$ Ryd (see Fig. 1), the effective He II Ly $\alpha$ line opacity due to absorption in the intergalactic diffuse gas can be estimated as $\tau_{\text {eff }}=\ln \left(I_{\mathrm{c}} / I_{\mathrm{GP}}\right)$.

Several absorption systems in the present work have neutral hydrogen column densities in the range $10^{16} \mathrm{~cm}^{-2}<N(\mathrm{HI})<$ $10^{17} \mathrm{~cm}^{-2}$, and thus are optically thin in the hydrogen Lyman continuum, but can be optically thick in the He II Lyman continuum. In Paper I we found that such systems can be described with an average ionizing spectrum which reflects both the external (He II GP absorption) and local effects (strong attenuation at $E>4$ Ryd due to He II continuum absorption in the cloud itself and an enhanced peak at $E=3$ Ryd due to recombining emission of He II Ly $\alpha$ ). An example of such average spectrum is plotted by the dashed line in Fig. 1.

Figure 2 shows ionization curves of carbon and silicon ions calculated for this spectrum in comparison to those calculated for the spectrum of HM $(z=3)$. The intensity depletion in the range $3<E<4$ Ryd strongly affects ionization fractions of carbon leading to enhanced ratios of $\mathrm{C}$ II/C IV and C III/C IV at $\log U \gtrsim-2.5$. The dependence of silicon ion fractions on $I_{\mathrm{GP}}$ is rather weak, but they are sensitive to photons with energies $E \leq 3 \mathrm{Ryd}-$ an enhanced intensity in this range leads to a higher $\mathrm{Si}$ IV/Si II ratio.

To complete this section, some words should be said about the evaluation of $\eta$. In the systems under study, we observe lines of neutral hydrogen, but we do not have spectral ranges with the corresponding He II lines. Nevertheless, assuming that all lines arise in the same gas and reconstructing the velocity and density distributions of the absorbing material from available hydrogen and metal line profiles, we are able to estimate the column density of single ionized helium as well. The ionization corrections of $\mathrm{He}$ II needed for this estimation are calculated using

1 In the present context we consider the GP effect as being caused by combined action of line blanketing and He II Ly $\alpha$ absorption in continuous intergalactic medium (true GP effect).

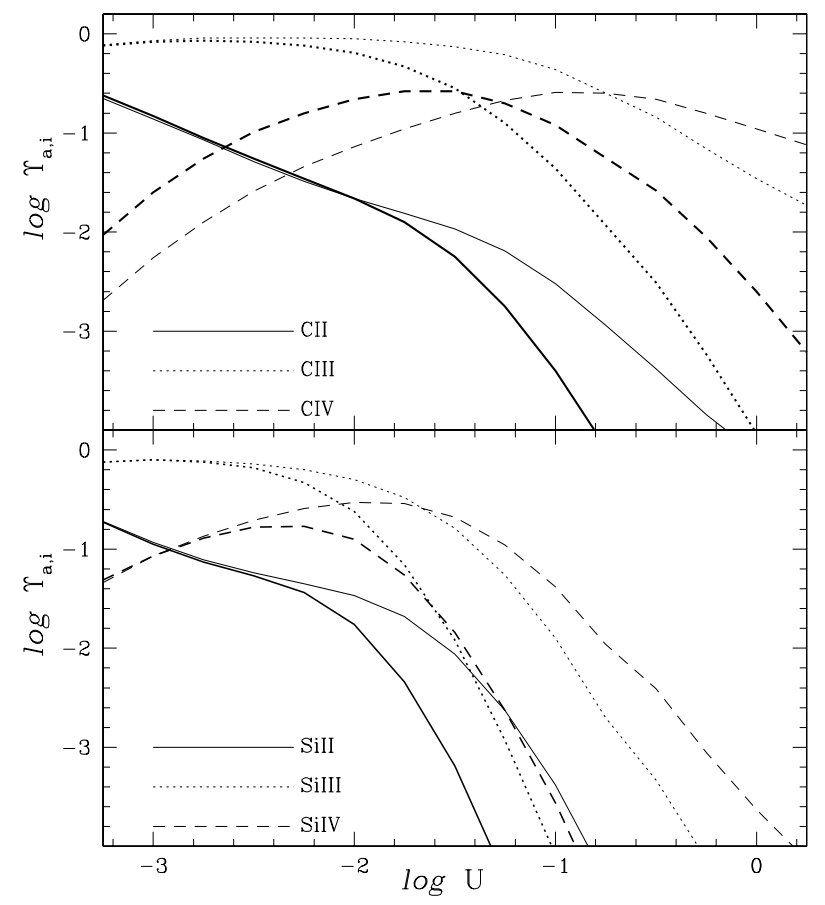

Fig. 2. Ionization fractions of carbon (upper panel) and silicon (lower panel) calculated for metallicity $Z=0.01 Z_{\odot}$. Thick curves correspond to the UBV shown by the solid line in Fig. 1, thin curves - to the $U V B$ shown by dashed line.

CLOUDY with the recovered $U V B$ spectral shape, metallicity and element abundance ratios as inputs.

\section{Analysis of individual absorption systems}

From a wealth of metal absorption systems identified in the spectra of HE 2347-4342, Q $1157+3143$, and HS $1700+6416$ only a few turned out to be suitable for the $U V B$ recovering. These metal absorption-line systems are described in detail in the subsections below.

In general, the uncertainties of the fitting parameters $U_{0}, N_{\mathrm{H}}$, $\sigma_{v}, \sigma_{y}$, and $Z_{a}$ listed in Tables $1-3$ are about $15-20 \%$ (for data with $S / N \gtrsim 30$ ).

All computations were performed with laboratory wavelengths and oscillator strengths taken from Morton (2003) for $\lambda>912 \AA$ and from Verner et al. (1994) for $\lambda<912 \AA$. Solar abundances were taken from Asplund et al. (2004). Note that their solar abundance of nitrogen, $N / H=6 \times 10^{-5}$, is 1.4 times (0.15 dex) lower then that previously reported in Holweger (2001).

The intensity $J_{912}$ needed to convert the obtained $U_{0}$ values into the gas density was taken from HM.

\subsection{Absorption systems towards HE 2347-4342}

We used for our analysis the VLT/UVES spectrum of HE 2347-4342. Observations and data processing are described in Shull et al. (2004).

\subsubsection{Absorption systems at $z=2.735,2.739$ and 2.741}

These three absorption systems have a joint column density of $N(\mathrm{H} \mathrm{I})=2.8 \times 10^{17} \mathrm{~cm}^{-2}$ (measured from Ly $\alpha$ discontinuity in the QSO spectrum). The system at $z_{\mathrm{abs}}=2.735$ (Fig. 3) exhibits 
Table 1. Physical parameters of the $z_{\mathrm{abs}}=2.735,2.739,2.741$ and 1.796 metal absorbers towards HE 2347-4342 derived by the MCISS procedure with the recovered UV background spectra shown in Fig. 4. Column densities are given in $\mathrm{cm}^{-2}$.

\begin{tabular}{|c|c|c|c|c|}
\hline $\begin{array}{l}\text { Parameter }^{a} \\
\text { (1) }\end{array}$ & $\begin{array}{c}z_{\mathrm{abs}}=2.735 \\
\quad(2)\end{array}$ & $\begin{array}{c}z_{\text {abs }}=2.739 \\
\text { (3) }\end{array}$ & $\begin{array}{c}z_{\mathrm{abs}}=2.741 \\
\quad(4)\end{array}$ & $\begin{array}{c}z_{\mathrm{abs}}=1.796 \\
(5)\end{array}$ \\
\hline$U_{0}$ & $3.3 \mathrm{E}-2$ & $4.4 \mathrm{E}-3$ & $4.3 \mathrm{E}-2$ & $2.0 \mathrm{E}-3$ \\
\hline$N_{\mathrm{H}}$ & 2.3E20 & 7.3E19 & $5.9 \mathrm{E} 20$ & $2.0 \mathrm{E} 17$ \\
\hline$\sigma_{\mathrm{v}}, \mathrm{km} \mathrm{s}^{-1}$ & 25.0 & 24.0 & 21.0 & 15.0 \\
\hline$\sigma_{\mathrm{y}}$ & 0.6 & 0.5 & 0.7 & 0.6 \\
\hline$Z_{\mathrm{C}}$ & $2.4 \mathrm{E}-6$ & $1.2 \mathrm{E}-6$ & $7.3 \mathrm{E}-8$ & $7.4 \mathrm{E}-4$ \\
\hline$Z_{\mathrm{N}}$ & $<2.0 \mathrm{E}-7$ & $\$ 8.0 \mathrm{E}-8$ & $\ldots$ & \\
\hline$Z_{\mathrm{O}}$ & $5.0 \mathrm{E}-6$ & $\ldots$ & $\ldots$ & $1.3 \mathrm{E}-3$ \\
\hline$Z_{\mathrm{Mg}}$ & $\ldots$ & $\ldots$ & $\ldots$ & $(1.4-2.5) \mathrm{E}-4$ \\
\hline$Z_{\mathrm{Al}}$ & & & & $1.1 \mathrm{E}-1$ \\
\hline$Z_{\mathrm{Si}}$ & $4.3 \mathrm{E}-7$ & $1.5 \mathrm{E}-7$ & $1.0 \mathrm{E}-8$ & $9.5 \mathrm{E}-5$ \\
\hline$Z_{\mathrm{Fe}}$ & $\ldots$ & $\ldots$ & $\ldots$ & $2.3 \mathrm{E}-5$ \\
\hline$\left[Z_{\mathrm{C}}\right]$ & -2.00 & -2.30 & -3.53 & 0.48 \\
\hline$\left[Z_{N}\right]$ & $<-2.5$ & $\lessgtr-2.87$ & $\ldots$ & \\
\hline$\left[Z_{\mathrm{O}}\right]$ & -2.00 & $\ldots$ & $\ldots$ & 0.45 \\
\hline$\left[Z_{\mathrm{Mg}}\right]$ & $\ldots$ & $\ldots$ & $\ldots$ & $0.60-0.85$ \\
\hline$\left[Z_{\mathrm{Al}}\right]$ & $\ldots$ & $\ldots$ & $\ldots$ & 0.6 \\
\hline$\left[Z_{\mathrm{Si}}\right]$ & $-1.90^{c}$ & $-2.34^{c}$ & $-3.53^{c}$ & 0.46 \\
\hline$\left[Z_{\mathrm{Fe}}\right]$ & $\ldots$ & $\ldots$ & $\ldots$ & -0.08 \\
\hline$N(\mathrm{H} \mathrm{I})$ & $(4.9 \pm 0.5) \mathrm{E} 16$ & $(8.6 \pm 1.0) \mathrm{E} 16$ & $(1.4 \pm 0.2) \mathrm{E} 17$ & $(9.1 \pm 2.0) \mathrm{E} 15$ \\
\hline$N(\mathrm{O} \mathrm{I})$ & $\ldots$ & $\ldots$ & $\ldots$ & $(1.0 \pm 0.2) \mathrm{E} 13$ \\
\hline$N(\mathrm{C}$ II $)$ & $(7.1 \pm 0.5) \mathrm{E} 12$ & $(4.1 \pm 0.4) \mathrm{E} 12$ & $(6.2 \pm 0.6) \mathrm{E} 11$ & $5.9 \mathrm{E} 13^{b}$ \\
\hline$N(\mathrm{Mg}$ II $)$ & $\ldots$ & & & $(0.9-1.6) \mathrm{E} 13$ \\
\hline$N(\mathrm{Si} \mathrm{II})$ & $(1.6 \pm 0.2) \mathrm{E} 12$ & $(5.5 \pm 0.5) \mathrm{E} 11$ & $(1.0 \pm 0.2) \mathrm{E} 11$ & $(1.4 \pm 0.1) \mathrm{E} 13$ \\
\hline$N(\mathrm{Fe}$ II $)$ & & & & $(9.4 \pm 0.5) \mathrm{E} 11$ \\
\hline$N(\mathrm{C}$ III $)$ & $(3.7 \pm 0.4) \mathrm{E} 14$ & $6.9 \mathrm{E} 13^{b}$ & $3.0 \mathrm{E} 13^{b}$ & $\ldots$ \\
\hline$N(\mathrm{~N}$ III $)$ & $\lesssim 2.5 \mathrm{E} 13$ & $\lesssim .6 \mathrm{E} 12$ & $\ldots$ & $\ldots$ \\
\hline$N(\mathrm{O}$ III $)$ & $(7.5 \pm 2.0) \mathrm{E} 14$ & $\ldots$ & $\ldots$ & $\ldots$ \\
\hline$N(\mathrm{Al}$ III $)$ & $\ldots$ & $\ldots$ & $\ldots$ & $(7.4 \pm 0.7) \mathrm{E} 11$ \\
\hline$N(\mathrm{Si}$ III $)$ & $(2.5 \pm 0.2) \mathrm{E} 13$ & $6.9 \mathrm{E} 12^{b}$ & $1.6 \mathrm{E} 12^{b}$ & $(4.9 \pm 1.0) \mathrm{E} 12$ \\
\hline$N(\mathrm{C}$ IV $)$ & $(1.2 \pm 0.1) \mathrm{E} 14$ & $(4.4 \pm 0.5) \mathrm{E} 12$ & $(9.5 \pm 1.5) \mathrm{E} 12$ & $(1.2 \pm 0.1) \mathrm{E} 13$ \\
\hline$N(\mathrm{Si} I V)$ & $(2.0 \pm 0.2) \mathrm{E} 13$ & $2.0 \pm 0.2) \mathrm{E} 12$ & $(1.1 \pm 0.1) \mathrm{E} 12$ & $(2.9 \pm 0.3) \mathrm{E} 12$ \\
\hline$\langle T\rangle, \mathrm{K}$ & $2.9 \mathrm{E} 4$ & $2.0 \mathrm{E} 4$ & $3.2 \mathrm{E} 4$ & $5.5 \mathrm{E} 3$ \\
\hline$n_{\mathrm{H}}, \mathrm{cm}^{-3}$ & $9.0 \mathrm{E}-4$ & $6.0 \mathrm{E}-3$ & $7.0 \mathrm{E}-4$ & $2.2 \mathrm{E}-2$ \\
\hline$L, \mathrm{kpc}$ & 90 & 4 & 280 & 0.004 \\
\hline$N(\mathrm{He}$ II $)$ & 7.4E $18^{b}$ & $4.3 \mathrm{E} 18^{b}$ & $2.0 \mathrm{E} 19^{b}$ & $2.0 \mathrm{E} 16^{b}$ \\
\hline & $150 \pm 20$ & $50 \pm 5$ & $140 \pm 20$ & $2 \pm 2$ \\
\hline$\tau_{\mathrm{GP}}^{\mathrm{He} \text { II }}$ (at 3 Ryd) & $1.45 \pm 0.2$ & & & 0 \\
\hline
\end{tabular}

all silicon and carbon transitions from single to triple ionized species, N III $\lambda 989 \AA$ (partially blended) and O III $\lambda 832 \AA$ (albeit from very noisy part of the spectrum). Expected positions of O VI $\lambda \lambda 1032,1037 \AA$ are blocked with Ly $\alpha$ forest absorptions. Test calculations with the HM ionizing spectrum ended up with synthetic line profiles which significantly overestimated the observed intensity of Si II and underestimated intensities of Si IV and C II. Such behavior of synthetic lines along with the procedure of spectral shape adjustment were already described in detail in Paper I, Sect. 3.1. The final spectral shape that we obtained is shown by the dashed line in Fig. 4 with corresponding physical parameters given in Table 1, Col. 2 and synthetic profiles plotted by the smooth lines in Fig. 3.

The adjusted ionizing spectrum is much softer at $E>4 \mathrm{Ryd}$ than the initial spectrum of HM and has a strong emission peak at $E=3$ Ryd (needed to reproduce the observed Si II/Si IV ratio). At the same time, the observed ratio of $\mathrm{C}$ II/C IV cannot be reproduced without a significant drop in the intensity between 3 and 4 Ryd. The obtained $U V B$ shape resembles that of $z_{\mathrm{abs}}=2.9171$ system towards HE 0940-1050 (Paper I, Sect. 3.1) and reflects both local (attenuation at $E>4$ Ryd and enhanced He II Ly $\alpha$ emission at $E=3$ Ryd) and external (GP depression at $3<E<4$ Ryd due to intergalactic He II Ly $\alpha$ absorption) effects. From the $I_{\mathrm{GP}}$ value the effective optical depth of the intergalactic He II Ly $\alpha$ absorption at $z_{\mathrm{abs}}=2.735$ is estimated as $\tau_{\mathrm{GP}}^{\mathrm{He} I I}=1.45 \pm 0.2$. The calculated He II column density is $7.4 \times 10^{18} \mathrm{~cm}^{-2}$ which gives $\eta=150$.

Two close absorption systems at $z_{\mathrm{abs}}=2.739$ and $z_{\mathrm{abs}}=2.741$ have metal lines either heavily blended or weak and noisy leading to the consequence that the observed profiles can be reproduced within a broad range of spectral shapes. In any case, the $U V B$ recovered from the $z_{\mathrm{abs}}=2.735$ system is consistent with these systems and delivers reasonable physical parameters. They are listed in Table 1, Cols. 2 and 3, with corresponding synthetic profiles plotted in Figs. 5 and 6 (smooth lines). Note an extremely low metallicity of $[Z] \simeq-3.5$ in the sub-LLS at $z_{\text {abs }}=$ 2.741 along with its huge linear size of $L>250 \mathrm{kpc}$. In spite of all uncertainties immanent to this absorber, these values can be 
Table 2. Physical parameters of the $z_{\text {abs }}=2.944,2.939$, and 2.875 metal absorbers towards Q $1157+3143$ derived by the MCISS procedure with the recovered UV background spectra shown in Fig. 9. Column densities are given in $\mathrm{cm}^{-2}$.

\begin{tabular}{|c|c|c|c|}
\hline $\begin{array}{l}\text { Parameter }^{a} \\
\text { (1) }\end{array}$ & $\begin{array}{c}z_{\text {abs }}=2.944 \\
\quad(2)\end{array}$ & $\begin{array}{c}z_{\text {abs }}=2.939 \\
\text { (3) }\end{array}$ & $\begin{array}{c}z_{\mathrm{abs}}=2.875 \\
(4)\end{array}$ \\
\hline$U_{0}$ & $1.6 \mathrm{E}-2$ & $8.8 \mathrm{E}-3$ & $6.9 \mathrm{E}-2$ \\
\hline$N_{\mathrm{H}}$ & 1.4E20 & $8.3 \mathrm{E} 18$ & 2.4E20 \\
\hline$\sigma_{\mathrm{v}}, \mathrm{km} \mathrm{s}^{-1}$ & 20.0 & 22.5 & 25.0 \\
\hline$\sigma_{\mathrm{y}}$ & 0.6 & 0.6 & 0.5 \\
\hline$Z_{\mathrm{C}}$ & $3.0 \mathrm{E}-7$ & $2.3 \mathrm{E}-5$ & $1.1 \mathrm{E}-6$ \\
\hline$Z_{\mathrm{N}}$ & $<3.0 \mathrm{E}-8$ & $<1.0 \mathrm{E}-6$ & \\
\hline$Z_{\mathrm{Si}}$ & $4.7 \mathrm{E}-8$ & $3.7 \mathrm{E}-6$ & $1.6 \mathrm{E}-7$ \\
\hline$\left[Z_{\mathrm{C}}\right]$ & -2.90 & -1.02 & -2.36 \\
\hline$\left[Z_{N}\right]$ & $<-3.3$ & $<-1.8$ & \\
\hline$\left[Z_{\mathrm{Si}}\right]$ & $-2.84^{d}$ & -0.94 & $-2.34^{d}$ \\
\hline$N(\mathrm{H} \mathrm{I})$ & $(1.1 \pm 0.2) \mathrm{E} 17$ & $1.2 \pm 0.1) \mathrm{E} 16$ & $(1.6 \pm 0.4) \mathrm{E} 16$ \\
\hline$N(\mathrm{C}$ II $)$ & $(1.4 \pm 0.2) \mathrm{E} 12$ & $(9.0 \pm 0.5) \mathrm{E} 12$ & $(2.0 \pm 0.3) \mathrm{E} 12$ \\
\hline$N(\mathrm{Si}$ II $)$ & $(2.5 \pm 0.2) \mathrm{E} 11$ & $(1.5 \pm 0.2) \mathrm{E} 12$ & $(1.8 \pm 0.2) \mathrm{E} 11$ \\
\hline$N(\mathrm{C}$ III $)$ & $(3.7 \pm 0.6) \mathrm{E} 13^{b}$ & $(1.8 \pm 0.2) \mathrm{E} 14$ & $(1.9 \pm 0.2) \mathrm{E} 14$ \\
\hline$N(\mathrm{~N}$ III $)$ & $<5.8 \mathrm{E} 12$ & $\lesssim 7.5 \mathrm{E} 12$ & 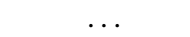 \\
\hline$N(\mathrm{Si}$ III $)$ & $(3.4 \pm 0.4) \mathrm{E} 12^{b}$ & $2.0 \mathrm{E} 13^{c}$ & $4.2 \mathrm{E} 12^{c}$ \\
\hline$N(\mathrm{C}$ IV $)$ & $(3.2 \pm 0.8) \mathrm{E} 12$ & $(1.1 \pm 0.3) \mathrm{E} 13$ & $(4.0 \pm 0.4) \mathrm{E} 13$ \\
\hline$N(\mathrm{Si}$ IV $)$ & $(1.7 \pm 0.3) \mathrm{E} 12$ & $(8.0 \pm 0.4) \mathrm{E} 12$ & $(6.8 \pm 0.5) \mathrm{E} 12$ \\
\hline$\langle T\rangle, \mathrm{K}$ & 2.7E4 & $1.8 \mathrm{E} 4$ & 3.7E4 \\
\hline$n_{\mathrm{H}}, \mathrm{cm}^{-3}$ & $2.0 \mathrm{E}-3$ & $3.0 \mathrm{E}-3$ & $4.0 \mathrm{E}-4$ \\
\hline$L, \mathrm{kpc}$ & 27 & 0.86 & 200 \\
\hline$N($ He II $)$ & $8.5 \mathrm{E} 18^{c}$ & $5.4 \mathrm{E} 17^{c}$ & $7.0 \mathrm{E} 18^{c}$ \\
\hline & $85 \pm 10$ & $45 \pm 5$ & $440 \pm 40$ \\
\hline$\tau_{\mathrm{GP}}^{\mathrm{Hell}}$ (at $3 \mathrm{Ryd}$ ) & \multicolumn{2}{|c|}{$2.5 \pm 0.3$} & $2.5-3$ \\
\hline
\end{tabular}

${ }^{a} Z_{\mathrm{X}}=N_{\mathrm{X}} / N_{\mathrm{H}} ;\left[Z_{\mathrm{X}}\right]=\log \left(N_{\mathrm{X}} / N_{\mathrm{H}}\right)-\log \left(N_{\mathrm{X}} / N_{\mathrm{H}}\right)_{\odot} ;{ }^{b}$ partially blended; ${ }^{c}$ calculated using the velocity-density distribution estimated from hydrogen and metal profiles; ${ }^{d}$ actual value may be 0.2 dex higher - see the last paragraph in Sect. 3.1.1.

considered as reliable: the $\mathrm{C}$ III/C IV ratio is similar to that found in the $z_{\mathrm{abs}}=2.735$ system (it implies the same mean ionization parameter and gas density), but there is three times more neutral hydrogen and an order of magnitude less C III, C IV and Si IV. As far as we know, this is the lowest metallicity ever directly measured in a $\operatorname{Ly} \alpha$ forest absorber.

An important result is that a significant difference in $\eta$ between the $z_{\mathrm{abs}}=2.739$ absorber $(\eta=50)$ and two other absorbers $(\eta \simeq 150)$ stems not from variations in the ionizing background (all quantities have been obtained with the same ionizing spectrum) but entirely from differences in the gas density: $n_{\mathrm{H}}=6.0 \times 10^{-3} \mathrm{~cm}^{-3}$ at $z_{\mathrm{abs}}=2.739$ and $(7-9) \times 10^{-4} \mathrm{~cm}^{-3}$ at $z_{\mathrm{abs}}=2.741$ and 2.735 (see also Fig. 19 below).

It is also worth noting that these three absorbers are located in the vicinity of the He II transmission window at $2.716<z<$ 2.726 marked by " $\mathrm{K}$ " in Figs. $1 f$ and 5 in Z04.

In all three systems we measured $[\mathrm{Si} / \mathrm{C}] \simeq 0-0.1$. In order to interpret this value correctly the following considerations should be taken into account. The system at $z_{\mathrm{abs}}=2.735$ used to restore the $U V B$ is optically thin in H I but opaque in He II which supposes that radiation transfer effects can be significant. The average spectrum is appropriate to describe the line profiles, but it can produce biased abundances. Computational experiments with different spectral shapes of the $U V B$ have shown that softening at $E>4$ Ryd affects more significantly the abundance of silicon making the recovered ratio $[\mathrm{Si} / \mathrm{C}]$ lower. In general, the change of $[\mathrm{Si} / \mathrm{C}]$ does not exceed $0.2 \mathrm{dex}$. This allows to set an upper bound to $[\mathrm{Si} / \mathrm{C}]$ in the these systems: $[\mathrm{Si} / \mathrm{C}] \lesssim 0.3$. This value is fully consistent with $[\mathrm{Si} / \mathrm{C}]$ measured recently in an extremely metal-poor DLA: $[\mathrm{C}]=-2.83 \pm 0.05,[\mathrm{Si}]=-2.57 \pm 0.05$ (Erni et al. 2006), and with measurements in metal-poor stars: $[\mathrm{Si} / \mathrm{C}]<0.3$ at $[\mathrm{Mg}]<-3$ (Cayrel et al. 2004).

The systems show a strong underabundance of nitrogen compared to the solar value: $[\mathrm{N} / \mathrm{C}]<-0.5$

\subsubsection{Absorption system at $z_{\mathrm{abs}}=1.7963$}

This system was firstly identified through its unusually strong Mg II lines (Fig. 7). It also contains absorption lines of O I $\lambda 1302 \AA, C$ II $\lambda 1334 \AA$ (blended), Si II $\lambda \lambda 1260,1193,1190$, 1526, and $1304 \AA$, Fe II $\lambda \lambda 2600,2382 \AA$, Al III $\lambda \lambda 1854,1862 \AA$, Si III $\lambda 1206 \AA$, C IV $\lambda 1548 \AA$, and Si IV $\lambda \lambda 1393,1402 \AA$. Unfortunately, positions of N V $\lambda \lambda 1238,1242 \AA$ coincide with Ly $\alpha$ forest absorptions, and both lines of the O VI doublet fall beyond the available wavelength coverage. From hydrogen lines, only a noisy $\operatorname{Ly} \alpha$ is present and its blue wing is blended. However, in spite of this uncertainty the available set of metal lines and especially the silicon transitions make it possible to estimate the shape of the underlying $U V B$ ionizing radiation.

Firstly, the O I $\lambda 1302 \AA$ line is observed together with C IV. $\mathrm{O}$ I traces neutral hydrogen and its ion fraction is practically independent on the $U V B$ spectral shape at $E>1$ Ryd. The presence of O I supposes the ionization parameter $U \leq 10^{-3}$, otherwise the fraction of neutral oxygen becomes too small and the resulting overabundance of oxygen to silicon or carbon would exceed 1 dex compared to solar values.

Secondly, all low ionization metal lines exhibit simple profiles and have the same $F W H M$ of $16 \mathrm{~km} \mathrm{~s}^{-1}$ (b-parameter equals $8.7 \mathrm{~km} \mathrm{~s}^{-1}$ ), which assumes turbulent motion as the main source of the line broadening. Even with this underestimated $b$-parameter (thermal component in the broadening of the hydrogen line is neglected) the neutral hydrogen column density of a few $10^{16} \mathrm{~cm}^{-2}$ leads already to a significant overestimation of the intensity in the unblended red wing. This means that the absorption system is optically thin in the hydrogen continuum and the self-shielding as a reason for the simultaneous presence of O I and C IV can be excluded. Thus, the only remaining option to observe a pronounced C IV absorption at $U \lesssim 10^{-3}$ is to have an ionizing spectrum which is very hard at $E>4$ Ryd.

Actually, such type of spectrum was recovered by us for the absorption system at $z_{\mathrm{abs}}=1.78$ towards HE 0141-3932 exhibiting a similar set of ions (Reimers et al. 2005a). So, it was naturally to take that spectral shape as an initial guess for the system under study. Test runs have shown that the observed intensities of silicon lines can be matched only with metallicities of 2.5-3 $Z_{\odot}$, otherwise the Si III line comes strongly overestimated for all tried spectral shapes. This behavior is explained by a strong dependence of the silicon ion fractions on temperature which at metallicities near and above solar overrides the dependence on spectral shape.

These considerations allow us to construct the $U V B$ spectrum appropriate for the $z_{\mathrm{abs}}=1.796$ absorption system (Fig. 4 , dotted line). Due to many uncertainties stated above this spectral shape is not unique. For example, the power index for the slope at $1<E<4$ Ryd can be higher or lower. This will mostly affect the ratio $\mathrm{C} / \mathrm{Si}$ making it above solar in case of a steeper slope. From this point of view a hard slope is preferable. However, the depth of the He II break at 4 Ryd can be constrained: intensities at $E=4$ Ryd below $0.06 J_{912}$ lead to a strong overabundance of oxygen to silicon and to carbon $([\mathrm{O} / \mathrm{Si}, \mathrm{C}]>0.2)$, and ought to be excluded. 
Table 3. Physical parameters of the $z_{\mathrm{abs}}=2.568,2.438,2.433,2.379$, and 1.845 metal absorbers towards HS $1700+6416$ derived by the MCISS procedure with the recovered UV background spectra shown in Fig. 13. Column densities are given in $\mathrm{cm}^{-2}$.

\begin{tabular}{|c|c|c|c|c|c|}
\hline $\begin{array}{l}\text { Parameter }{ }^{a} \\
\text { (1) }\end{array}$ & $\begin{array}{c}z_{\text {abs }}=2.568 \\
\text { (2) }\end{array}$ & $\begin{array}{c}z_{\text {abs }}=2.438 \\
\text { (3) }\end{array}$ & $\begin{array}{c}z_{\mathrm{abs}}=2.433 \\
\quad(4)\end{array}$ & $\begin{array}{c}z_{\mathrm{abs}}=2.379 \\
(5)\end{array}$ & $\begin{array}{c}z_{\mathrm{abs}}=1.845 \\
(6)\end{array}$ \\
\hline$U_{0}$ & $1.3 \mathrm{E}-1$ & $1.8 \mathrm{E}-2$ & $9.4 \mathrm{E}-3$ & $1.1 \mathrm{E}-1$ & $7.9 \mathrm{E}-3$ \\
\hline$N_{\mathrm{H}}$ & 3.3E18 & $1.2 \mathrm{E} 19$ & 2.0E19 & 3.2E19 & 2.1E19 \\
\hline$\sigma_{\mathrm{v}}, \mathrm{km} \mathrm{s}^{-1}$ & 20.5 & 17.0 & 23.0 & 27.0 & 35.0 \\
\hline$\sigma_{\mathrm{y}}$ & 0.5 & 0.5 & 0.7 & 0.6 & 0.6 \\
\hline$Z_{\mathrm{C}}$ & $2.0 \mathrm{E}-5$ & $9.2 \mathrm{E}-6$ & $1.5 \mathrm{E}-5$ & $2.8 \mathrm{E}-6$ & $8.4 \mathrm{E}-5$ \\
\hline$Z_{N}$ & $\lesssim 3.0 \mathrm{E}-6$ & $\lesssim 2.0 \mathrm{E}-6$ & $\ldots$ & & $1.7 \mathrm{E}-5$ \\
\hline$Z_{\mathrm{O}}$ & $1.0 \mathrm{E}-4$ & $\ldots$ & $\ldots$ & $1.6 \mathrm{E}-5$ & $2.5 \mathrm{E}-5$ \\
\hline$Z_{\mathrm{Mg}}$ & $\ldots$ & $\ldots$ & $\ldots$ & $\ldots$ & $2.5 \mathrm{E}-5$ \\
\hline$Z_{\mathrm{Al}}$ & $\ldots$ & $\ldots$ & $\ldots$ & $\ldots$ & $1.0 \mathrm{E}-6$ \\
\hline$Z_{\mathrm{Si}}$ & $6.2 \mathrm{E}-6$ & $1.8 \mathrm{E}-6$ & $2.4 \mathrm{E}-6$ & $8.4 \mathrm{E}-7$ & $1.4 \mathrm{E}-5$ \\
\hline$\left[Z_{C}\right]$ & -1.08 & -1.42 & -1.20 & -1.94 & -0.46 \\
\hline$\left[Z_{N}\right]$ & $\lesssim-1.3$ & $\lesssim-1.5$ & $\ldots$ & & -0.55 \\
\hline$\left[Z_{\mathrm{O}}\right]$ & -0.65 & $\ldots$ & $\ldots$ & -1.52 & -0.25 \\
\hline$\left[Z_{\mathrm{Mg}}\right]$ & $\ldots$ & $\ldots$ & $\ldots$ & $\ldots$ & -0.25 \\
\hline$\left[Z_{\mathrm{Al}}\right]$ & $\ldots$ & $\ldots$ & $\ldots$ & $\ldots$ & -0.36 \\
\hline$\left[Z_{\mathrm{Si}}\right]$ & -0.75 & -1.24 & -1.12 & -1.61 & -0.36 \\
\hline$N(\mathrm{H} \mathrm{I})$ & $2.5 \mathrm{E} 14^{b}$ & $(3.6 \pm 1.5) \mathrm{E} 15$ & $(2.6 \pm 1.0) \mathrm{E} 16$ & $(2.4 \pm 0.3) \mathrm{E} 15$ & $2.5 \mathrm{E} 16^{b}$ \\
\hline$N(\mathrm{C}$ II $)$ & $(1.4 \pm 0.2) \mathrm{E} 11$ & $(1.3 \pm 0.3) \mathrm{E} 12$ & $(1.3 \pm 0.2) \mathrm{E} 13$ & $\ldots$ & $3.6 \mathrm{E} 13^{c}$ \\
\hline$N(\mathrm{Mg}$ II $)$ & $\ldots$ & $\ldots$ & $\ldots$ & $\ldots$ & $2.2 \mathrm{E} 12$ \\
\hline$N(\mathrm{Si}$ II $)$ & $\ldots$ & $\$ 3.0 \mathrm{E} 11$ & $(2.2 \pm 0.2) \mathrm{E} 12$ & & $(3.9 \pm 0.2) \mathrm{E} 12$ \\
\hline$N(\mathrm{C}$ III $)$ & $(1.2 \pm 0.2) \mathrm{E} 13$ & $(6.7 \pm 0.6) \mathrm{E} 13$ & $(2.5 \pm 0.3) \mathrm{E} 14$ & $(2.3 \pm 0.4) \mathrm{E} 13$ & $\ldots$ \\
\hline$N(\mathrm{~N}$ III $)$ & $\ldots$ & $\lesssim 1.4 \mathrm{E} 13$ & $\ldots$ & $\ldots$ & $\ldots$ \\
\hline$N(\mathrm{O}$ III $)$ & $\ldots$ & $\ldots$ & $\ldots$ & $\ldots$ & $1.6 \mathrm{E} 15^{c}$ \\
\hline$N(\mathrm{Al}$ III $)$ & $\ldots$ & $\ldots$ & $\ldots$ & $\ldots$ & $(5.8 \pm 0.6) \mathrm{E} 11$ \\
\hline$N(\mathrm{Si}$ III $)$ & $(4.2 \pm 0.3) 1 \mathrm{E} 11$ & $(5.0 \pm 0.3) \mathrm{E} 12$ & $(2.8 \pm 0.2) \mathrm{E} 13$ & $(6.7 \pm 0.3) \mathrm{E} 11$ & $3.5 \mathrm{E} 13^{d}$ \\
\hline$N(\mathrm{C}$ IV $)$ & $(1.2 \pm 0.1) \mathrm{E} 13$ & $(2.5 \pm 0.3) \mathrm{E} 13$ & $(3.6 \pm 0.2) \mathrm{E} 13$ & $(1.6 \pm 0.1) \mathrm{E} 13$ & $(4.3 \pm 0.2) \mathrm{E} 14$ \\
\hline$N(\mathrm{O}$ IV $)$ & $\ldots$ & (20-5 & $\ldots$ & $\ldots$ & $1.8 \mathrm{E} 15^{c}$ \\
\hline$N(\mathrm{Si}$ IV $)$ & $(4.8 \pm 0.4) \mathrm{E} 11$ & $(4.0 \pm 0.2) \mathrm{E} 12$ & $(1.2 \pm 0.1) \mathrm{E} 13$ & $(5.3 \pm 0.5) \mathrm{E} 11$ & $(3.8 \pm 0.3) \mathrm{E} 13$ \\
\hline$N(\mathrm{~N} \mathrm{v})$ & & 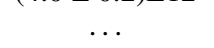 & $\ldots$ & & $(3.5 \pm 0.3) \mathrm{E} 13$ \\
\hline$N(\mathrm{O} \mathrm{VI})$ & $(3.9 \pm 0.4) \mathrm{E} 13$ & $\ldots$ & $\ldots$ & $(3.0 \pm 0.3) \mathrm{E} 13$ & $\ldots$ \\
\hline$\langle T\rangle, \mathrm{K}$ & $3.3 \mathrm{E} 4$ & $2.6 \mathrm{E} 4$ & $2.1 \mathrm{E} 4$ & $4.3 \mathrm{E} 4$ & $1.5 \mathrm{E} 4$ \\
\hline$n_{\mathrm{H}}, \mathrm{cm}^{-3}$ & $2.5 \mathrm{E}-4$ & $1.5 \mathrm{E}-3$ & $3.0 \mathrm{E}-3$ & $3.0 \mathrm{E}-4$ & $4.0 \mathrm{E}-3$ \\
\hline$L, \mathrm{kpc}$ & 4.0 & 3 & 2 & 38 & 2 \\
\hline$N(\mathrm{He}$ II $)$ & $2.6 \mathrm{E} 16^{c}$ & $3.7 \mathrm{E} 17^{c}$ & $1.1 \mathrm{E} 18^{c}$ & $2.9 \mathrm{E} 17^{c}$ & $3.5 \mathrm{E} 17^{c}$ \\
\hline & $100 \pm 10$ & $100 \pm 10$ & $45 \pm 5$ & $120 \pm 20$ & $16 \pm 5$ \\
\hline$\tau_{\mathrm{GP}}^{\mathrm{He} I \mathrm{II}}$ (at $3 \mathrm{Ryd}$ ) & $1.2 \pm 0.2$ & \multicolumn{2}{|c|}{$1.2 \pm 0.2$} & $1.2 \pm 0.2$ & 0 \\
\hline
\end{tabular}

${ }^{a} Z_{\mathrm{X}}=N_{\mathrm{X}} / N_{\mathrm{H}} ;\left[Z_{\mathrm{X}}\right]=\log \left(N_{\mathrm{X}} / N_{\mathrm{H}}\right)-\log \left(N_{\mathrm{X}} / N_{\mathrm{H}}\right)_{\odot} ;{ }^{b}$ column density calculated from the velocity-density distribution estimated from metal profiles assuming constant metallicity within the absorber; ${ }^{c}$ calculated using the velocity and density distributions derived from hydrogen and metal profiles; ${ }^{d}$ partially blended.

Physical parameters estimated with the $U V B$ from Fig. 4 are given in Table 1, Col. 5. Strong relative underabundance of iron (three times solar values for carbon, oxygen and silicon and only 0.8 for iron) can be considered as reliable since it was reproduced for all tried spectral shapes. Aluminium is slightly overabundant compared to $\mathrm{C}, \mathrm{O}$ and $\mathrm{Si}$ (4 times solar), but the accuracy of this estimation is not high enough due to uncertainties in the recombination coefficients used in CLOUDY. As for magnesium, a spread of its abundance (4-7 solar) is explained by the saturation of $\mathrm{Mg}$ II $\lambda 2796 \AA$ and possible contamination of Mg II $\lambda 2803 \AA$ with a telluric line.

\subsection{Absorption systems towards Q $1157+3143$}

Absorption spectrum of this quasar was obtained with Keck/HIRES. Details on the data reduction are given in Kirkman \& Tytler (1999). Additionally, this bright quasar was observed with HST/STIS to study He II Ly $\alpha$ absorption in the intergalactic matter (Reimers et al. 2005b).

\subsubsection{Absorption system at $z_{\mathrm{abs}}=2.944$}

This absorption system (Fig. 8) lies very close to the quasar $\left(z_{\mathrm{em}}=2.97\right.$, Ly $\alpha$ forest starts at $\left.z=2.999\right)$ and could be associated. However, Q 1157+3143 does not show proximity effect. On the contrary, the line density in its vicinity is unusually high (Ganguly et al. 2001). A set of ions detected in the $z_{\mathrm{abs}}=2.944$ system is also typical for an intergalactic absorber: associated systems are usually highly ionized and show pronounced lines of OVI, NV, and CIV along with weak C III, Si III, and S IV, whereas the system under study reveals C III and Si III lines stronger than C IV, Si IV, and no N V absorption. At the expected position of the O VI $\lambda 1031 \AA$ line only a weak absorption is seen (its identification as O VI is ambiguous since O VI $\lambda 1037 \AA$ is blended).

From the H I continuum absorption in the observed spectrum the neutral hydrogen column density of this system (together with its neighbor at $\left.z_{\mathrm{abs}}=2.939\right)$ is estimated as $(1.5 \pm 0.2) \times$ $10^{17} \mathrm{~cm}^{-2}$. Such a high hydrogen content together with rather weak intensities of the metal lines point to a very low metallicity 


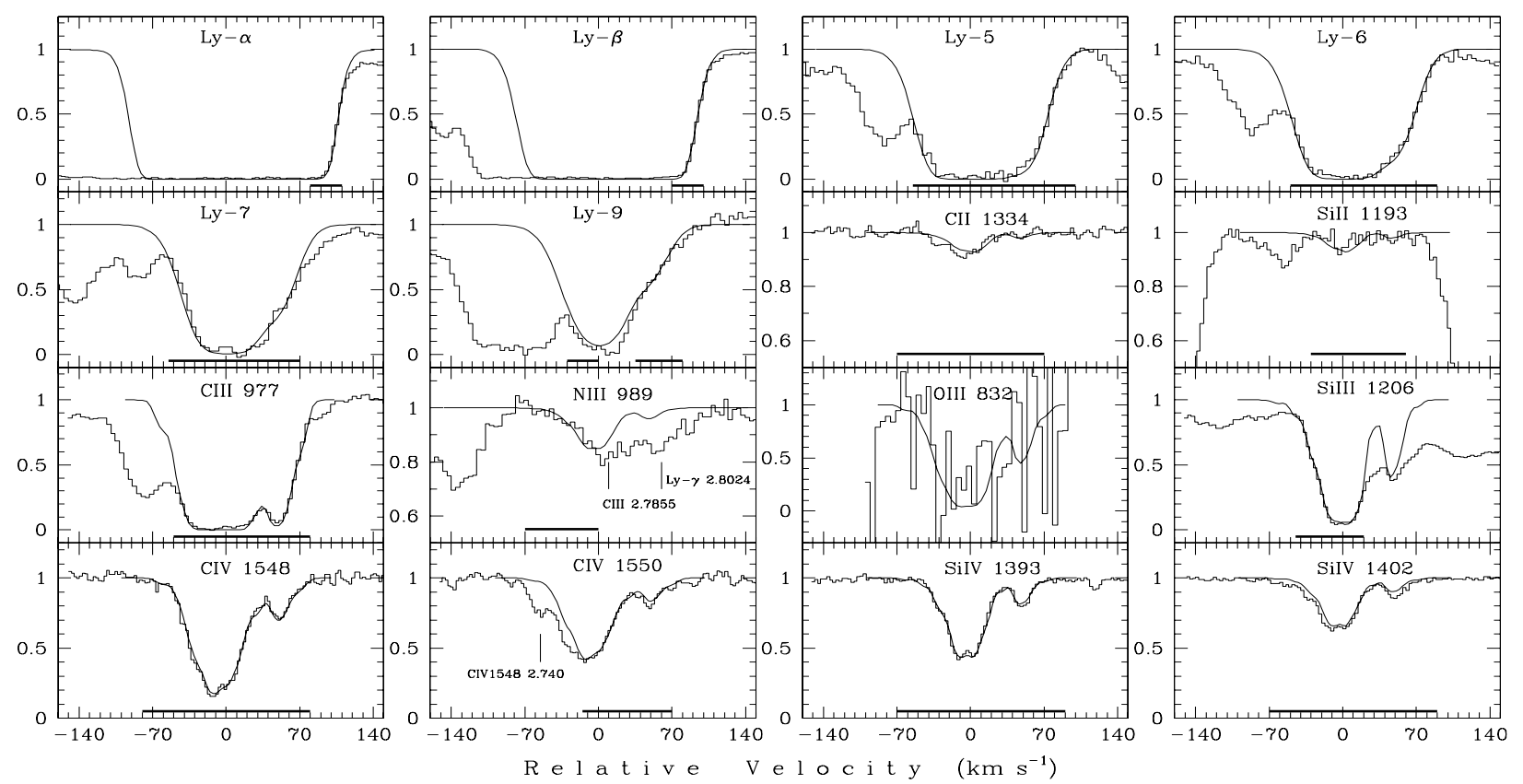

Fig. 3. Hydrogen and metal absorption lines associated with the $z_{\text {abs }}=2.735$ system towards HE $2347-4342$ (histograms). Synthetic profiles corresponding to the recovered ionizing spectrum (dashed line in Fig. 4) are plotted by the smooth curves. The physical parameters are listed in Table 1, Col. 2. Bold horizontal lines mark pixels included in the objective function of the MCI procedure. The zero radial velocity is fixed at $z=2.73576$. The central positions of blends are indicated by tick marks.

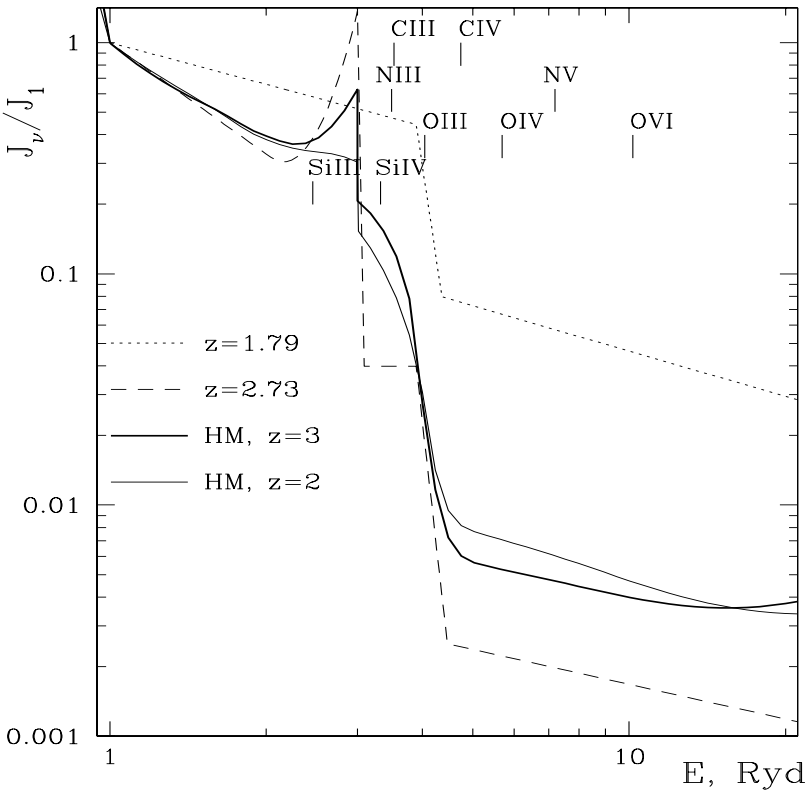

Fig. 4. The ionizing background spectrum predicted for $z=3$ and $z=2$ in $\mathrm{HM}$ (thick and thin solid lines, respectively) and the spectra restored for $z=2.73$ (dashed lines) and $z=1.79$ (dotted line). The normalization is the same as in Fig. 1.

of the absorbing gas which is another argument against the associated nature of the $z_{\mathrm{abs}}=2.944$ system.

Test calculations with the HM ionizing spectrum confirmed the metallicity of $0.001 Z_{\odot}$. However, with this spectrum the calculated intensity of the $\mathrm{C}$ III line in the range $-70<v<$ $70 \mathrm{~km} \mathrm{~s}^{-1}$ was significantly less than observed. Since the position of this line is in the $\operatorname{Ly} \alpha$ forest, possible blends should be taken into account. We do not find any metal absorption contaminating the $\mathrm{C}$ III line. The only candidate for blending could be a hydrogen absorption at $z_{\mathrm{abs}}=2.17$. If we assume that observed profile is mainly caused by this hydrogen absorption, then the kinetic temperature of the gas estimated from the line width is $T_{\text {kin }} \leq 32000 \mathrm{~K}$. This implies that the ionization parameter $U$ is less than 0.02 (for metallicity $[Z]<-1.5$, since there are no metal lines detected at $z_{\mathrm{abs}}=2.17$ ) and the linear size of the putative H I absorber does not exceed $0.5 \mathrm{kpc}$. Such small cloud would hardly survive in the IGM. Another argument in favor of C III is the comparison with the Si III line: both lines have similar widths $\left(F W H M=40 \mathrm{~km} \mathrm{~s}^{-1}\right.$ and $\left.36.5 \mathrm{~km} \mathrm{~s}^{-1}\right)$ and the same asymmetry. Thus, we can assume with high probability that the absorption at $-70<v<70 \mathrm{~km} \mathrm{~s}^{-1}$ is indeed caused by $\mathrm{C}$ III from the $z_{\mathrm{abs}}=2.944$ system. In this case only a spectrum with a significant flux depression between 3 and 4 Ryd makes it possible to fit adequately all observed lines.

The reconstructed spectral shape of the $U V B$ is shown in Fig. 9 by the dotted line, the corresponding physical parameters are given in Table 2, Col. 2, and the synthetic profiles are plotted by the solid lines in Fig. 8 .

Here we find again a spectrum similar to that from the $z_{\mathrm{abs}}=$ 2.9171 system (HE 0940-1050, Paper I): its shape reflects both the external (GP depression) and local effects (an enhanced emission peak at 3 Ryd and a sharp break at 4 Ryd) arising from the reprocession of the incident ionizing radiation by the absorbing cloud itself.

The He II Ly $\alpha$ opacity is estimated as $\tau_{\mathrm{GP}}^{\mathrm{He} I \mathrm{I}}=2.5 \pm 0.2$. The predicted value for the He II column density is $8.5 \times 10^{18} \mathrm{~cm}^{-2}$ which gives $\eta=85$.

The ratio $[\mathrm{Si} / \mathrm{C}]=0.06$, but as mentioned in Sect. 3.1.1 the actual value may be 0.2 dex higher: $[\mathrm{Si} / \mathrm{C}] \lesssim 0.26$.

A significant relative underabundance of nitrogen, $[\mathrm{N} / \mathrm{C}]<$ -0.3 , is also worth noting for this system. 


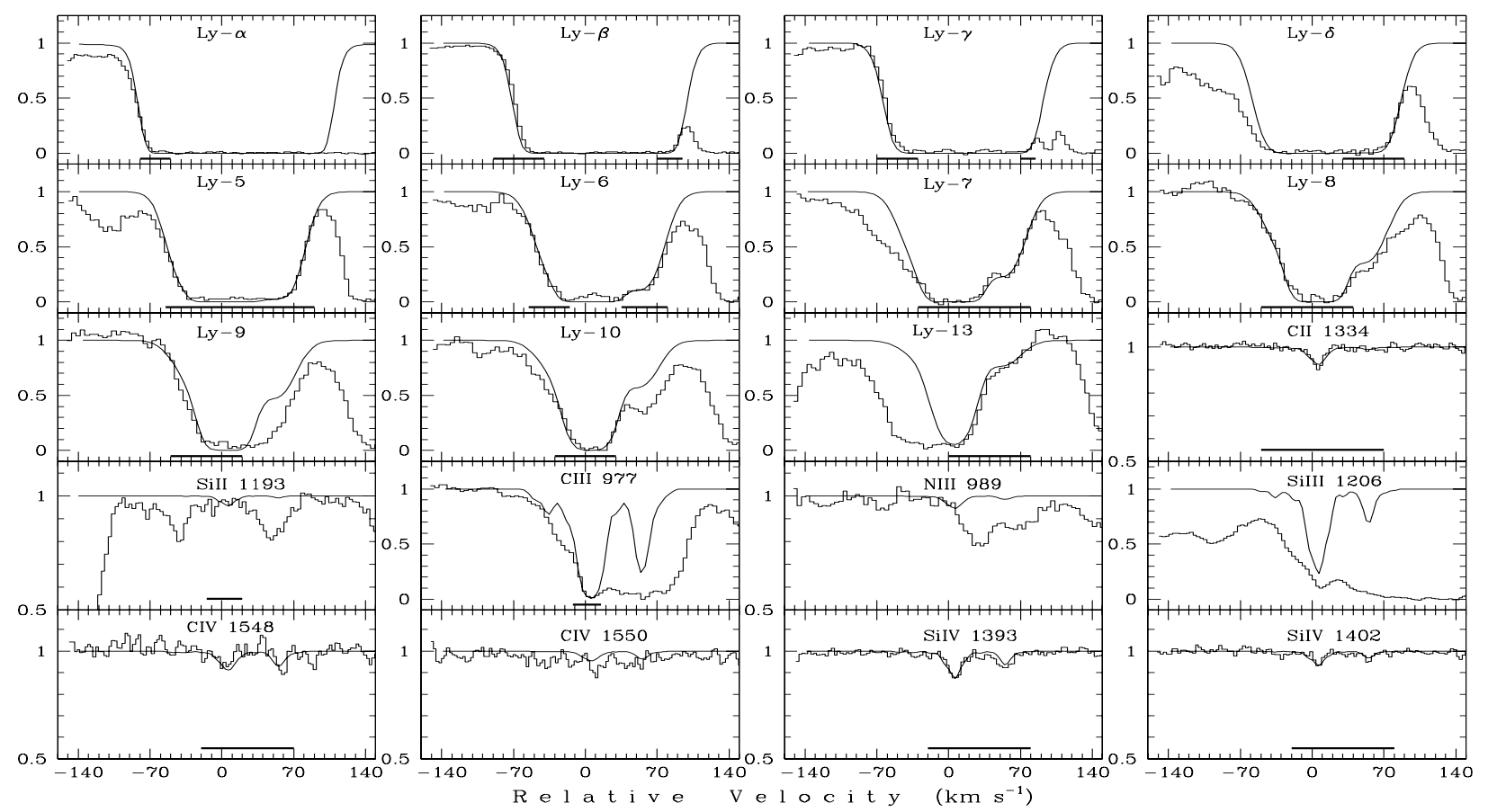

Fig. 5. Same as Fig. 3 but for the $z_{\text {abs }}=2.739$ system (HE 2347-4342). The zero radial velocity is fixed at $z=2.73914$. The estimated physical parameters are listed in Table 1, Col. 3.
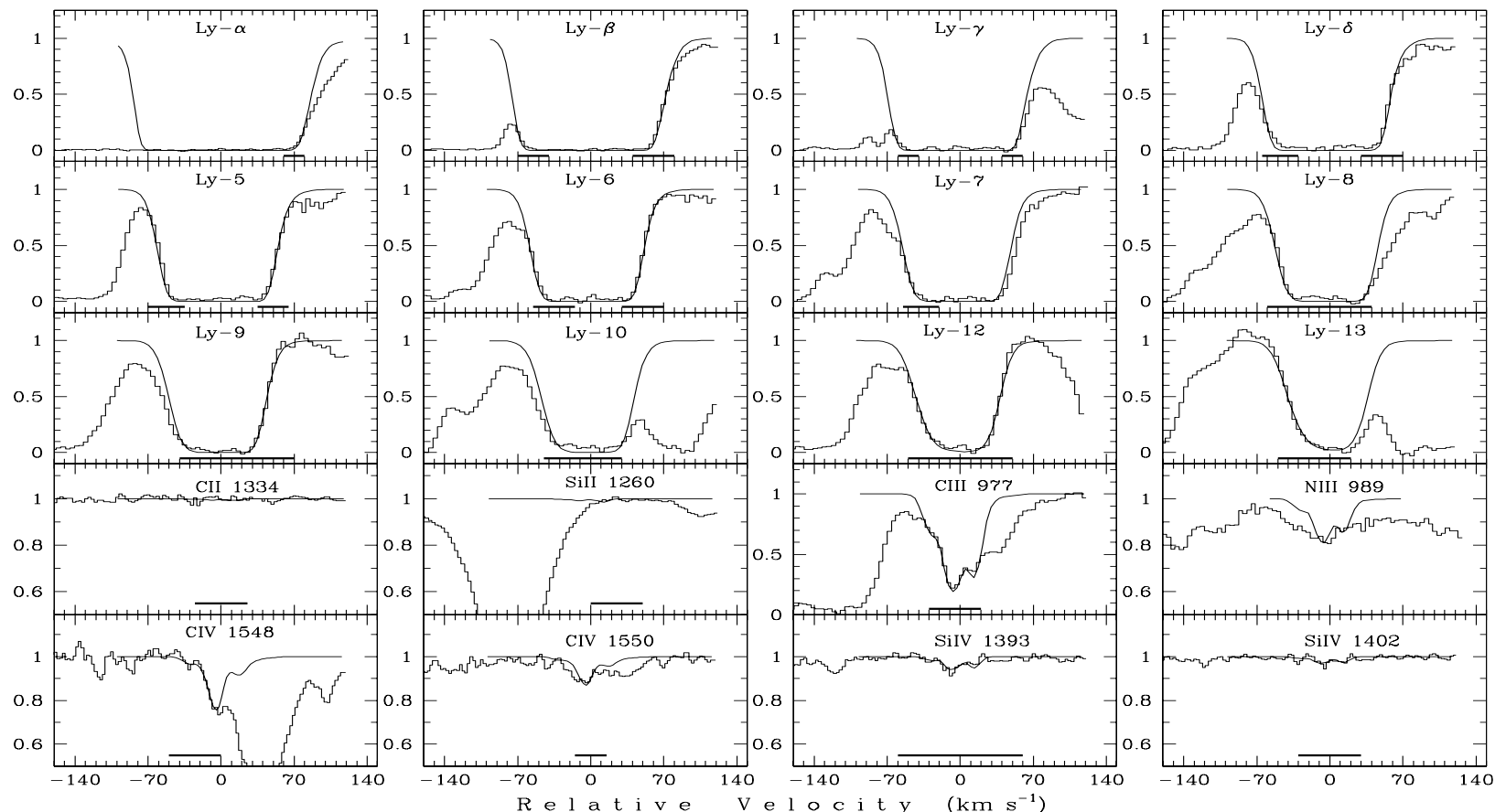

Fig. 6. Same as Fig. 3 but for the $z_{\mathrm{abs}}=2.741$ system (HE 2347-4342). The zero radial velocity is fixed at $z=2.74097$. The estimated physical parameters are listed in Table 1, Col. 4.

\subsubsection{Absorption system at $z_{\mathrm{abs}}=2.939$}

This system presents metal lines of both low (C II, Si II) and high (CIV, Si IV) ionization stages (Fig. 10). Lines O VI $\lambda \lambda 1031,1037 \AA$ are blended with strong Ly $\alpha$ forest absorptions. The spectrum of HM turned out to be inconsistent with the observed line intensities, the adjusted spectral shape is shown in Fig. 9 by the short-dashed line. The corresponding physical parameters are given in Table 2, Col. 3, and the synthetic profiles are plotted by the solid lines in Fig. 10.
Although shifted by only $\Delta z=0.0045\left(\Delta v=344 \mathrm{~km} \mathrm{~s}^{-1}\right)$ from the $z_{\mathrm{abs}}=2.944$ system, the present absorber reveals significantly different physical conditions: metallicity two order of magnitude higher along with a lower ionization parameter (higher gas density) and a very small (for an intergalactic absorber) linear size below $1 \mathrm{kpc}$.

The $z_{\text {abs }}=2.939$ system has optical depth $\simeq 1$ in the He II Lyman continuum. Thus, the attenuation of the incident radiation is probably not very pronounced and the recovered 


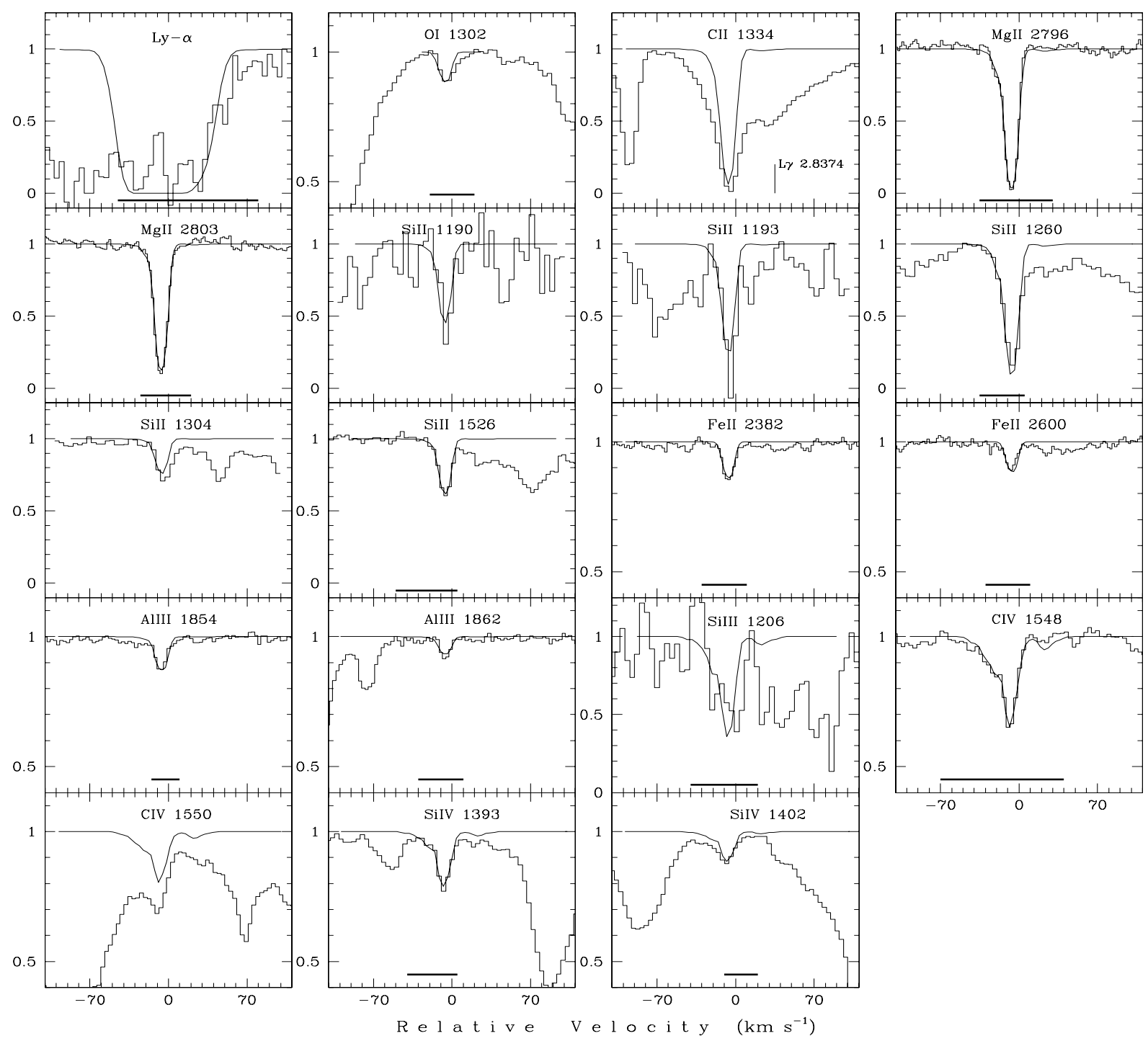

Fig. 7. Same as Fig. 3 but for the $z_{\text {abs }}=1.796$ system (HE 2347-4342). Synthetic profiles (smooth curves) were calculated with the recovered ionizing spectrum shown by the dotted line in Fig. 4. The zero radial velocity is fixed at $z=1.7963$. The estimated physical parameters are listed in Table 1, Col. 5. Synthetic profiles of Mg II are calculated for the abundance $2.5 \times 10^{-4}$ (see text). Blends are indicated by tick marks.

spectral shape can represent in this case the external $U V B$. It is seen from Fig. 9 that spectrum is softer at $E>4$ Ryd than the model spectrum of HM and has a more pronounced He II Ly $\alpha$ emission peak at $E=3$ Ryd. This is probably a consequence of a high number density of clouds registered at $z \lesssim 3$ along the sight line towards Q $1157+3143$.

The predicted value of $\eta=45 \pm 5$ is two times lower than $\eta$ in the $z_{\mathrm{abs}}=2.944$ system and reflects both the higher gas density and the ionizing background which is harder at $E>4$ Ryd (see Fig. 9).

Noteworthy is again a very strong relative underabundance of nitrogen, $[\mathrm{N} / \mathrm{C}]<-0.8$.

\subsubsection{Absorption system at $z_{\mathrm{abs}}=2.875$}

Hydrogen and metal lines identified in this system are shown in Fig. 11. The C IV $\lambda 1548 \AA$ line falls in a gap between echelle orders, N III $\lambda 989 \AA$ and O VI $\lambda \lambda 1031,1037 \AA$ are severely blended. Note an extremely weak Si II $\lambda 1260 \AA$ line which is nevertheless very well detected owing to high $S / N=150$ in this wavelength range.
Test calculations with the HM ionizing spectrum showed carbon content $[\mathrm{C} / \mathrm{H}]=-2.3$ associated with a very high relative overabundance of silicon, $[\mathrm{Si} / \mathrm{C}] \simeq 0.8$. The calculated intensity at the expected position of C III came significantly underestimated, but blending cannot be excluded (the only candidate H I Ly $\alpha$ at $z_{\mathrm{abs}}=2.115$ ).

The hydrogen column density is not well defined because of high noise at the locations of Ly-7 $\lambda 926 \AA$ and Ly- $8 \lambda 923 \AA$, but, in any case, it does not exceed $3 \times 10^{16} \mathrm{~cm}^{-2}$.

The obtained overabundance of silicon points to the ionizing spectrum much softer at $E>4$ Ryd than the HM spectrum that we used. A chance that high $[\mathrm{Si} / \mathrm{C}]$ arises from contribution of the hypothetical Pop III stars with masses $M \geq 100 M_{\odot}$ (pairinstability $\mathrm{SNe}$ ) seems quite unprobable since such overabundance has never been observed even in the extremely metal-poor clouds with $Z \lesssim 0.001 Z_{\odot}$ (see Sect. 3.1.1). A soft spectrum can be produced either by the He II continuous absorption (both in the IGM and inside the cloud itself) or by the input of radiation from soft sources like stars. In the present case the first option is preferable taking into account the observational evidence of large amount of He II at $z_{\mathrm{abs}} \simeq 2.87$ towards Q $1157+3143$ 


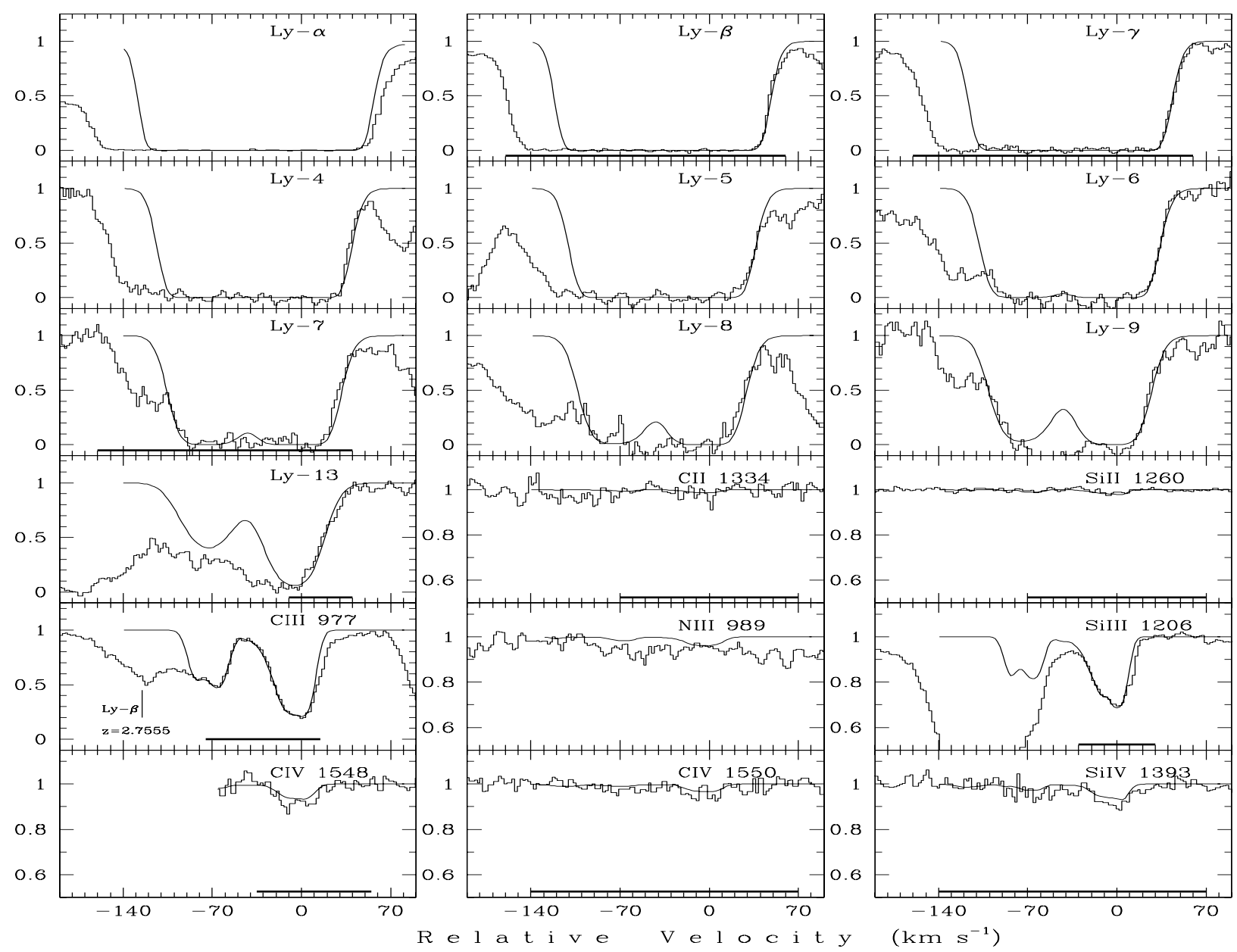

Fig. 8. Same as Fig. 3 but for the $z_{\text {abs }}=2.944$ system towards Q 1157+3143. Synthetic profiles (smooth curves) were calculated with the recovered ionizing spectrum shown by the dotted line in Fig. 9. The zero radial velocity is fixed at $z=2.9444$. The estimated physical parameters are listed in Table 2, Col. 2. Blends are indicated by tick marks.

(Reimers et al. 2005b), and a high value of the ionization parameter $U(\log (\mathrm{Si} \mathrm{IV} / \mathrm{Si} \mathrm{II}) \simeq 1.6)$ which supposes $\eta>150$ (see Fig. 19) which assusmes, in turn, that the cloud is opaque in He II.

Unfortunately, high noise in the C II $\lambda 1334 \AA$ profile and blending of Si III hamper the accurate reconstruction of the ionizing background. Calculations with the spectrum from the $z_{\mathrm{abs}}=2.944$ system lead to the silicon overabundance $[\mathrm{Si} / \mathrm{C}] \simeq$ $0.2-0.3$. Taking into account that the average spectrum gives [Si/C] by $\lesssim 0.2$ dex lower than the real value, this translates to $[\mathrm{Si} / \mathrm{C}] \approx 0.4-0.5$ which is slightly higher than measured elsewhere (Sect. 3.1.1). A $U V B$ with a lowered by 0.2 dex depression between 3 and 4 Ryd (Fig. 9, long-dashed line) gives $[\mathrm{Si} / \mathrm{C}] \simeq 0$ (and reproduces the observed line intensity at the position of C III). These two shapes constrain the acceptable range. The physical parameters obtained with the latter spectrum are listed in Table 2, Col. 4. The corresponding synthetic profiles are plotted by the solid lines in Fig. 11. The calculated column density of He II, $N(\mathrm{He}$ II $)=7.0 \times 10^{18} \mathrm{~cm}^{-2}$, supposes that the cloud is optically thick in the He II continuum and, thus, the restored spectral shape is again a superposition of the local and external effects.

The mean optical depth of He II Ly $\alpha, \tau_{\mathrm{GP}}^{\mathrm{He} I I}=2.5-3$, is comparable to $\tau_{\mathrm{GP}}^{\mathrm{He} I \mathrm{I}}=2.09 \pm 0.1$ estimated at $z_{\mathrm{abs}}=2.87$ by Reimers et al. (2005b).

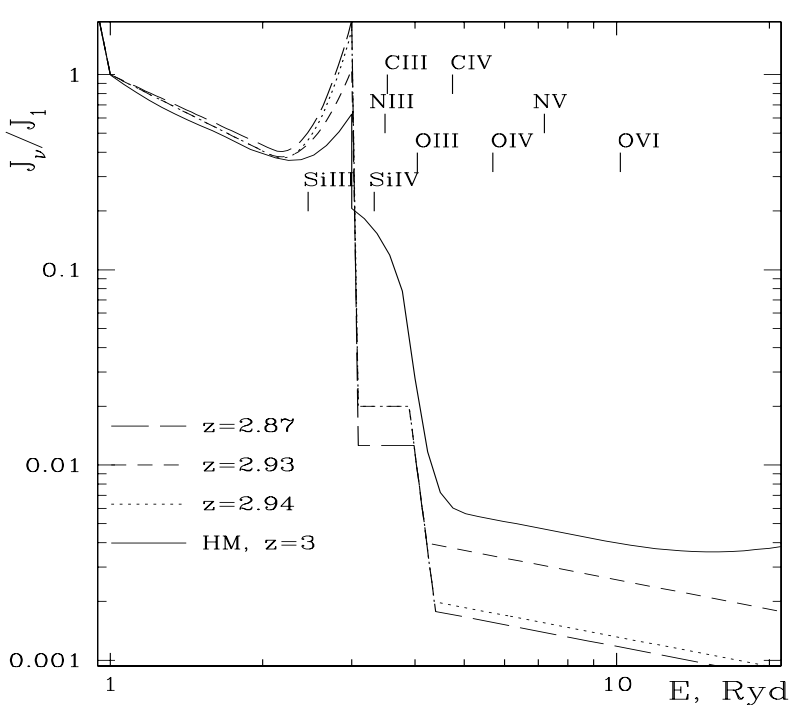

Fig. 9. Same as Fig. 4 but for the systems at $z_{\text {abs }}=2.944,2.939$, and 2.875 towards Q $1157+3143$.

We note the extremely high value of $\eta=440 \pm 40$ which is reliably reproduced in all test runs in spite of uncertainties in both the neutral hydrogen content and the spectral shape of the 


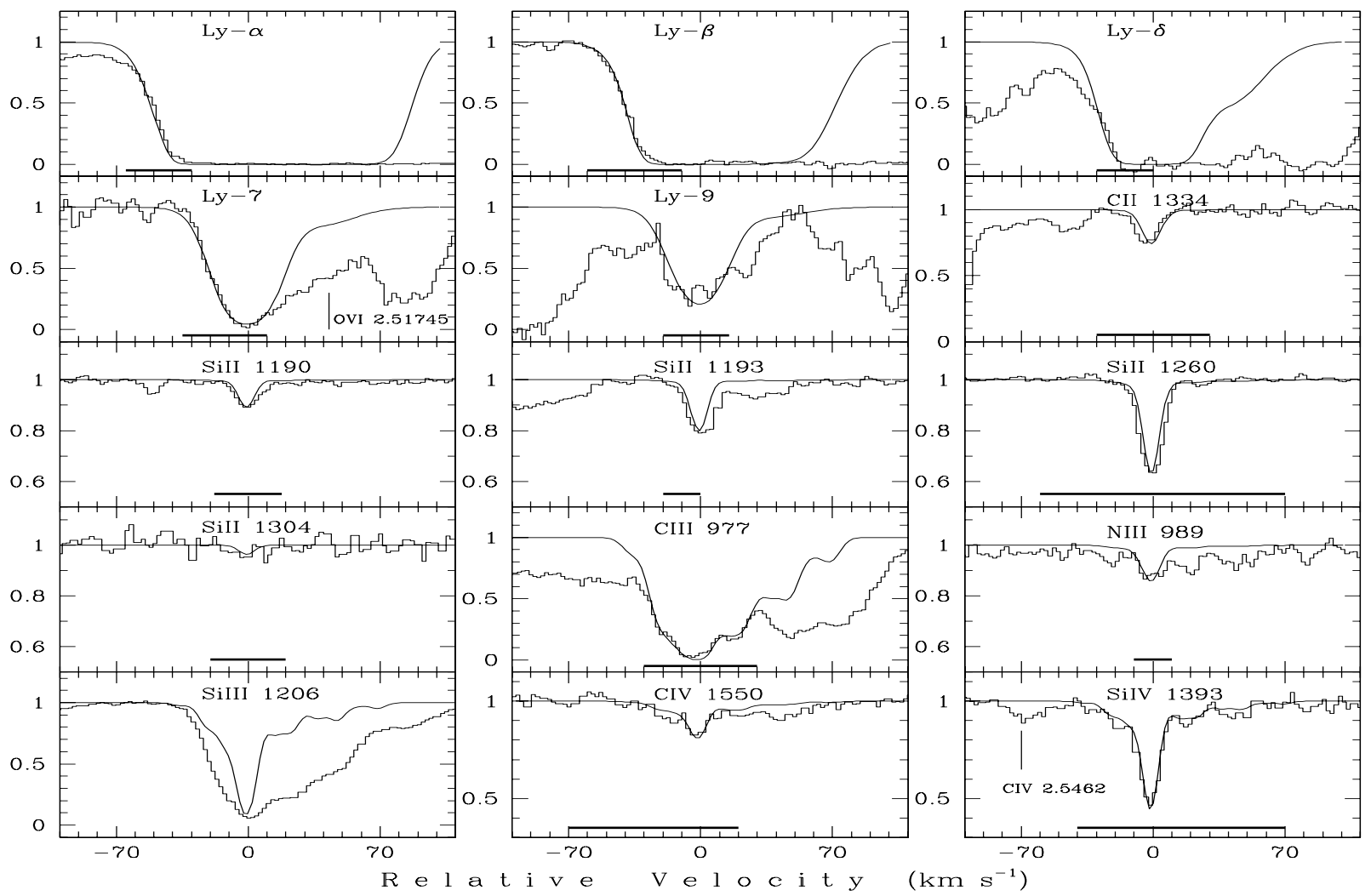

Fig. 10. Same as Fig. 3 but for the $z_{\text {abs }}=2.939$ system towards Q 1157+3143. Synthetic profiles (smooth curves) were calculated with the recovered ionizing spectrum shown by the short-dashed line in Fig. 9. The zero radial velocity is fixed at $z=2.93987$. The estimated physical parameters are listed in Table 2, Col. 3. Blends are indicated by tick marks.
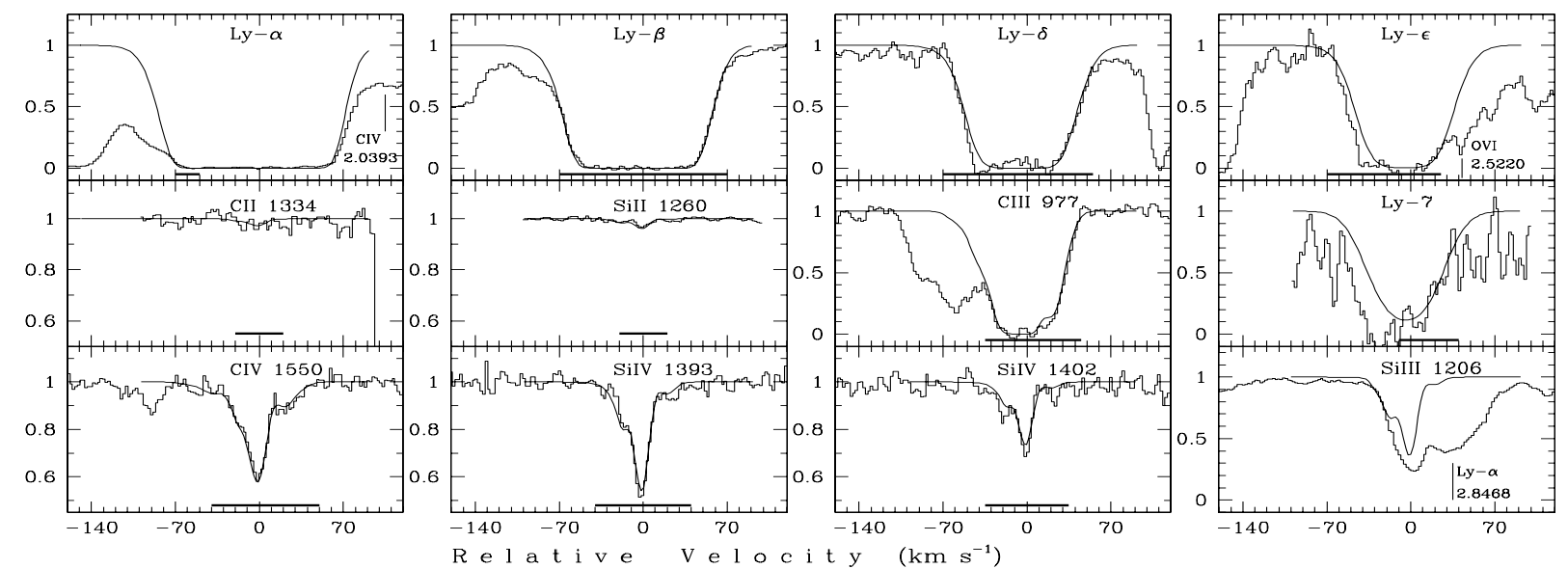

Fig. 11. Same as Fig. 3 but for the $z_{\text {abs }}=2.875$ system towards Q $1157+3143$. Synthetic profiles (smooth curves) were calculated with the recovered ionizing spectrum shown by the long-dashed line in Fig. 9 . The zero radial velocity is fixed at $z=2.87567$. The estimated physical parameters are listed in Table 2, Col. 4. Blends are indicated by tick marks.

ionizing radiation. This value can be explained by the softness of the effective ionizing radiation field and by the low gas density in the absorbing cloud (cf. with $\eta=150$ at $z_{\text {abs }}=2.944$ produced by the ionizing spectrum of similar softness).

\subsection{Absorption systems towards HS $1700+6416$}

This one of the brightest known high redshift quasar was observed many times with many instruments, both ground- and satellite-based. Reimers et al. (1992) and Vogel \& Reimers (1995) firstly reported on 7 strong metal absorption systems identified in its low-resolution combined optical and UV spectra. Later on, Davidsen et al. (1996) measured opacity of the intergalactic He II Ly $\alpha$ using far UV spectrum obtained with the HUT. Simcoe et al. (2002, 2006) studied physical conditions in the metal absorption systems using highresolution Keck telescope data. A comprehensive analysis of both HI and He II (FUSE) spectra was performed by F06 and Fechner \& Reimers (2007). For our reconstruction of the underlying $U V B$ spectra we selected systems with many different ionic lines, that are in the ionization equilibrium and do 

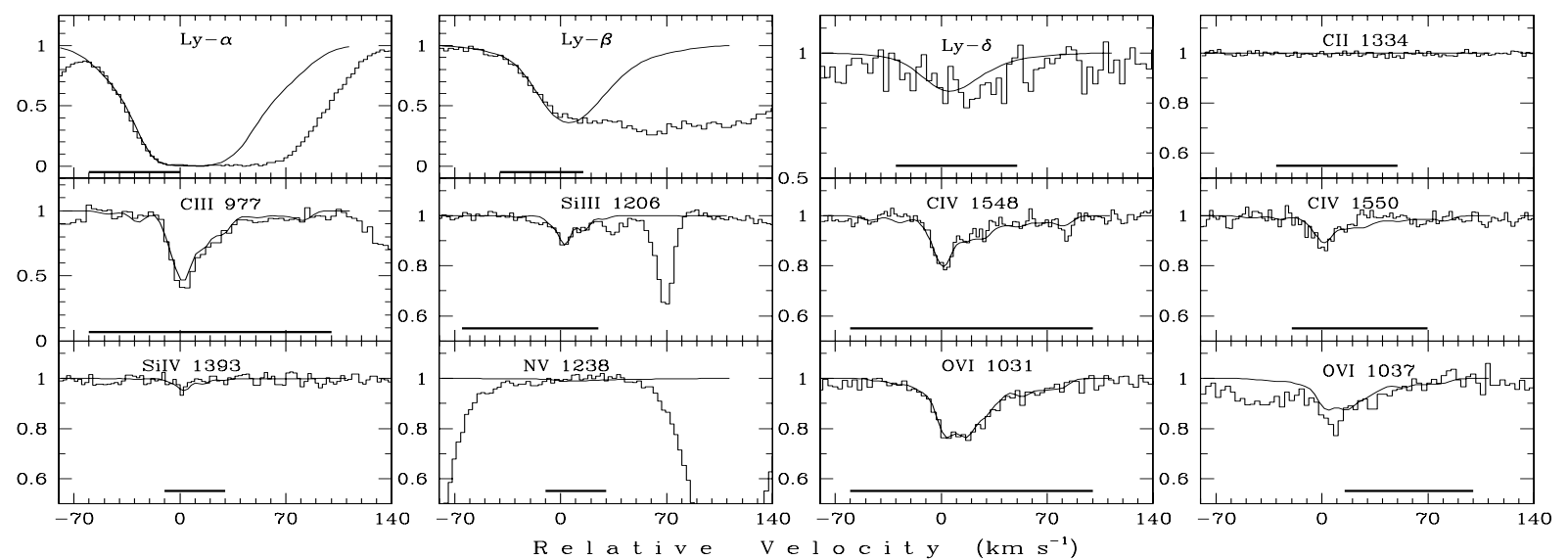

Fig. 12. Same as Fig. 3 but for the $z_{\text {abs }}=2.568$ system towards HS $1700+6416$. Synthetic profiles (smooth curves) were calculated with the recovered ionizing spectrum shown by the dotted line in Fig. 13. The zero radial velocity is fixed at $z=2.56826$. The estimated physical parameters are listed in Table 3, Col. 2.

not show self-shielding effects ${ }^{2}$. Observational data stem from the Keck/HIRES $(\Delta \lambda=3150-9000 \AA)$ and HST/STIS $(\Delta \lambda=$ $1155-1700 \AA)$ spectra. Details on data reduction are given in Simcoe et al. (2002) and Fechner et al. (2006b).

\subsubsection{Absorption system at $z_{\mathrm{abs}}=2.568$}

Hydrogen and metal lines identified in this system are shown in Fig. 12. Test calculations with the ionizing spectrum of HM revealed that the red wing of $\mathrm{HI} \mathrm{Ly} \alpha$ is inconsistent with the assumption of constant metallicity inside the absorber. In the following, the red wings of hydrogen lines are reconstructed from the density-velocity distributions derived from metal lines under the assumption of constant metallicity.

Calculations with the HM spectrum produced also strongly underestimated intensity of C III. Blending of C III seems rather unprobable since the line is weak, narrow and its asymmetric profile is similar to those of C IV and O VI. Thus the shape of the HM ionizing spectrum was modified. The result is shown in Fig. 13 by the dotted line. The corresponding physical parameters are given in Table 3, Col. 2, and the synthetic profiles are plotted by the solid lines in Fig. 12.

The mean optical depth of the intergalactic He II Ly $\alpha$ absorption decreases at redshift $z_{\mathrm{abs}}=2.56$ to the value $\tau_{\mathrm{GP}}^{\mathrm{He} \text { II }}=$ $1.2 \pm 0.2$. Note again a significant underabundance of nitrogen, $[\mathrm{N} / \mathrm{C}]<-0.3$.

\subsubsection{Absorption systems at $z_{\mathrm{abs}}=2.438$ and $z_{\mathrm{abs}}=2.433$}

Both absorption systems (Figs. 14 and 15) are parts of a large absorption complex stretching over $1000 \mathrm{~km} \mathrm{~s}^{-1}$ (see Fig. 6 in Simcoe et al. 2006) and represent dense clumps embedded in

${ }^{2}$ Since metal systems towards HS $1700+6416$ are well known, the reader may be interested in more details why other systems were not included in the $U V B$ recovering procedure: $z=2.578$ - two overlapping systems with different metallicities which cannot be clearly separated; $z=2.315$ - probably a non-equilibrial system with overionized O VI, may be associated with an AGN/QSO close to the line of sight; $z=2.168-$ the ratios $\mathrm{Si}$ IV/Si II $\simeq 40$ and $\mathrm{Si} \mathrm{IV} / \mathrm{C}$ IV $\simeq 0.16$ point out to a very soft $U V B$ (cf. data for the $z=2.875$ system in Table 2), however, uncertainties in C II and Si III (blending?) and the absence of C III $\lambda 977 \AA$ do not allow us to reconstruct the actual spectral shape; $z=1.724-$ most metal lines are contaminated by the Ly $\alpha$ forest absorptions.

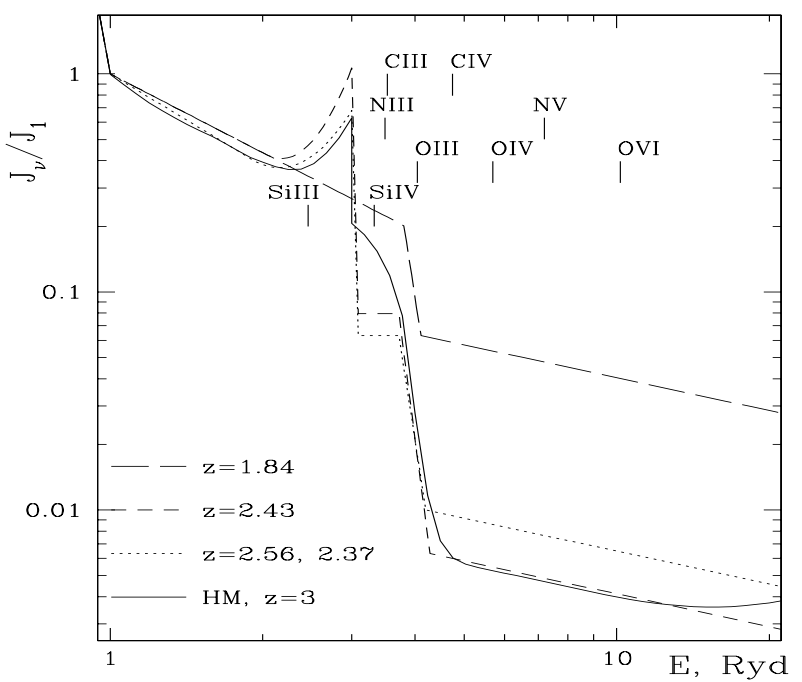

Fig. 13. Same as Fig. 4 but for the systems at $z_{\mathrm{abs}}=2.568,2.433,2.438$, 2.379 , and 1.845 towards HS $1700+6416$.

a highly ionized gas traced by O VI. While this highly ionized gas could be still non-equilibrial, the systems under consideration have already reached the ionization equilibrium with the background radiation owing to their higher gas density and, hence, a shorter recombination time. Note that the whole absorbing complex resembles the $z_{\mathrm{abs}}=1.8073$ complex towards HS 0747+4259 described in Sect. 4.1 in Paper II.

From low-resolution spectrum, the neutral hydrogen column density of the whole complex is estimated as $4.0 \times 10^{16} \mathrm{~cm}^{-2}$ (Vogel \& Reimers 1995). Test calculations with the HM ionizing spectrum resulted in an underestimated intensity of C II $\lambda 1334 \AA$ along with a strong overestimation of Si II $\lambda 1193 \AA$. The adjusted spectral shape is shown in Fig. 13 by the short-dashed line. The calculated physical parameters are listed in Table 3, Cols. 3 and 4 , and the corresponding synthetic profiles are shown by the solid lines in Figs. 14 and 15.

Although the spectral shape differs from that recovered for the $z_{\mathrm{abs}}=2.568$ system, the mean He II optical depth is the same: $\tau_{\mathrm{GP}}^{\mathrm{He} I I}=1.2 \pm 0.2$. This value is to be compared with $\tau_{\mathrm{GP}}^{\mathrm{He} \mathrm{II}}=1.0 \pm$ 0.07 measured at $z=2.4$ in the low resolution far UV spectrum of HS $1700+6416$ by Davidsen et al. (1996), and with $\tau_{\mathrm{GP}}^{\mathrm{He}}=$ $0.74 \pm 0.34$ at $z=2.45$ measured from FUSE data by F06. It 

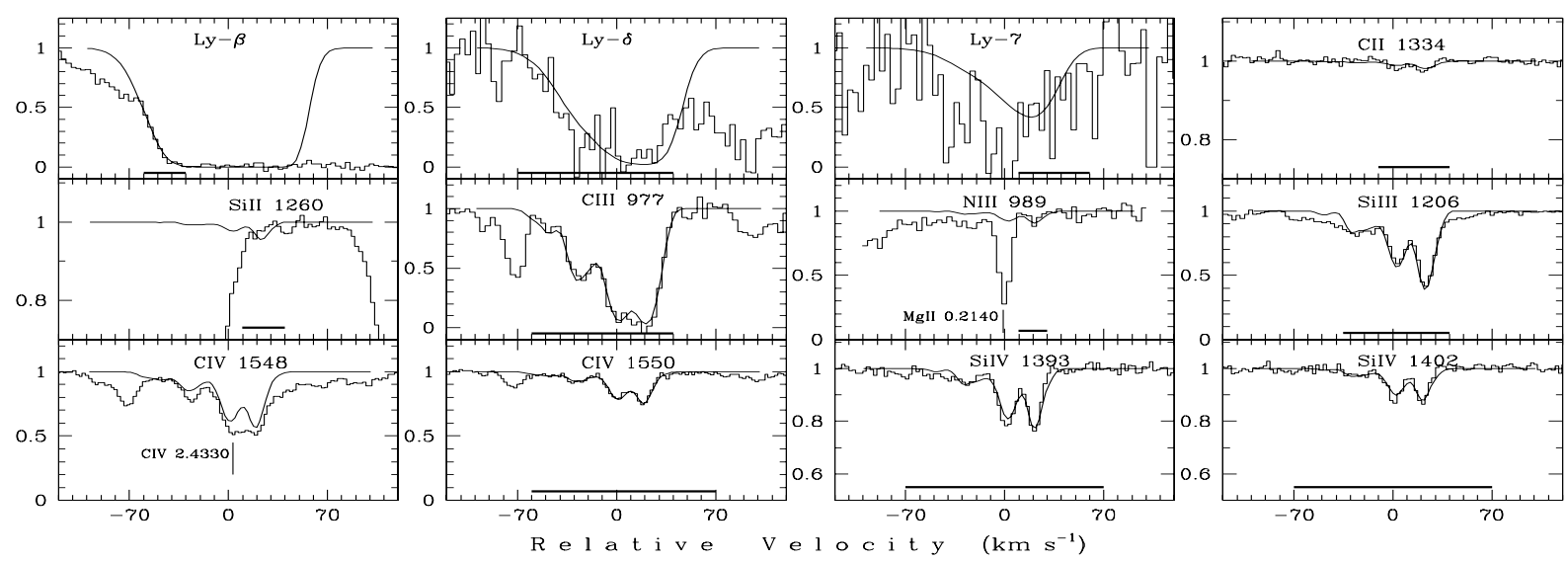

Fig. 14. Same as Fig. 3 but for the $z_{\text {abs }}=2.438$ system towards HS $1700+6416$. Synthetic profiles (smooth curves) were calculated with the recovered ionizing spectrum shown by the short-dashed line in Fig. 13. The zero radial velocity is fixed at $z=2.4386$. The estimated physical parameters are listed in Table 3, Col. 3. Blends are indicated by tick marks.

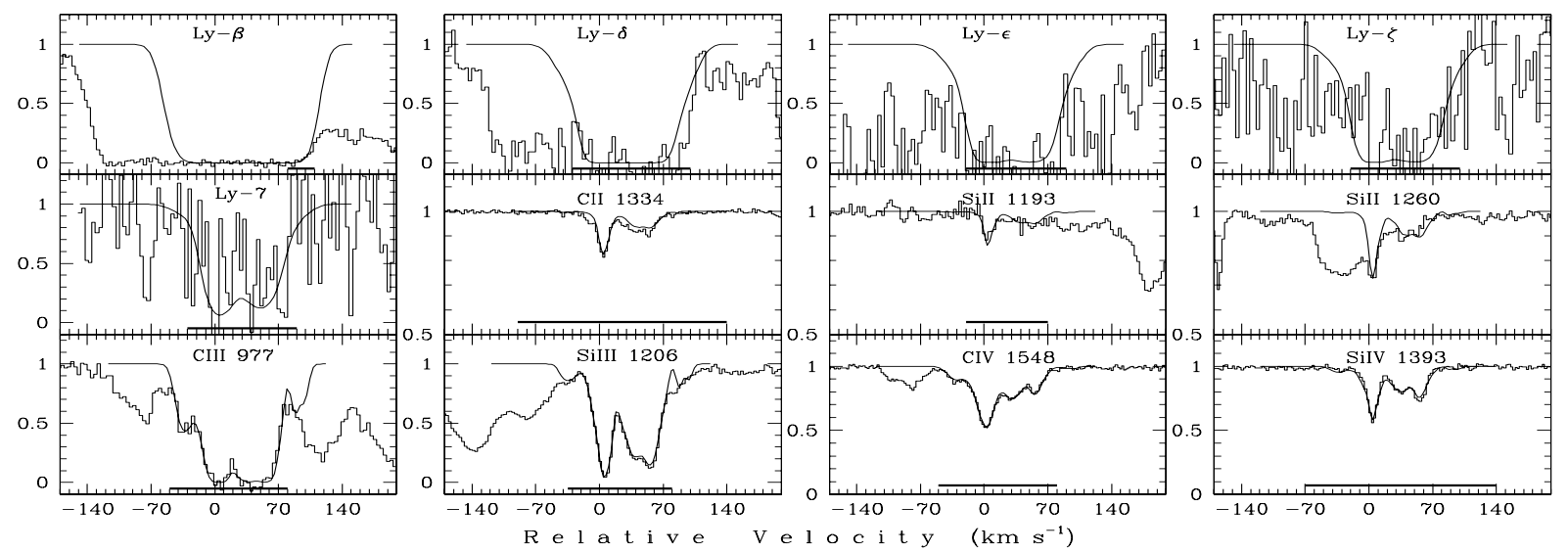

Fig. 15. Same as Fig. 3 but for the $z_{\text {abs }}=2.433$ system towards HS $1700+6416$. Synthetic profiles (smooth curves) were calculated with the recovered ionizing spectrum shown by the short-dashed line in Fig. 13. The zero radial velocity is fixed at $z=2.4330$. The estimated physical parameters are listed in Table 3, Col. 4.

should be noted, however, that latter value can be underestimated due to problems with zero level flux in the FUSE spectrum (see Sect. 2 in F06).

Another point worth noting is the variation we find in $\eta: \eta=$ 45 at $z_{\mathrm{abs}}=2.433$ and $\eta=100$ at $z_{\mathrm{abs}}=2.438$ (Table 3 ), which occurs because of the density differences in the clouds under study.

The value of $\eta$ at $z_{\mathrm{abs}}=2.438$ is close to the $\eta$ estimated for the previous $z_{\mathrm{abs}}=2.568$ system although the gas density in these two absorbers is quite different. This result is a consequence of softer $U V B$ at $z_{\mathrm{abs}}=2.438$ (see Fig. 19 in Sect. 4).

\subsubsection{Absorption system at $z_{\text {abs }}=2.379$}

A set of metal lines observed in this system (Fig. 16) is identical to that from the $z_{\mathrm{abs}}=2.568$ absorber described in Sect. 3.3.1. Unfortunately, a noisy profile of C III hampers the accurate reconstruction of the spectral shape of the underlying radiation. Although this line lies deep in the Ly $\alpha$ forest, it is very narrow $\left(F W H M=22.5 \mathrm{~km} \mathrm{~s}^{-1}\right)$ and cannot be a hydrogen line. On the other hand, no metal candidate for possible blending was found. Thus, the observed absorption is with high probability due to C III. However, the spectrum of HM underestimates the observed intensity of C III far above the noise level, whereas the spectrum derived for the $z_{\mathrm{abs}}=2.433$ system underestimates Si III. At the same time, the spectral shape from $z_{\mathrm{abs}}=2.568$ system turned out to be quite consistent with all observed intensities. The physical parameters obtained with this $U V B$ are given in Table 3, Col. 5 with the corresponding synthetic profiles shown by the solid lines in Fig. 16.

\subsubsection{Absorption system at $z_{\mathrm{abs}}=1.845$}

This system reveals one hydrogen line ( $\operatorname{Ly} \alpha)$ and a lot of metals in different ionic transitions (Fig. 17) which allow us to probe the underlying ionizing background. The column density of neutral hydrogen was estimated from low resolution spectrum by Vogel \& Reimers $(1995)$ as $5.6 \times 10^{16} \mathrm{~cm}^{-2}$, i.e. this system is optically thin in the hydrogen continuum. It is thin in the He II Lyman continuum as well: the attenuation of the incident radiation due to continuous He II absorption in the cloud itself leads to an increasing Si IV/C IV ratio, but we find in this system $\mathrm{Si}$ IV/C IV $=0.088$ which is well below the values measured in the systems with local attenuations at $z_{\mathrm{abs}}=2.735,2.944$, and 2.875 (Tables 1 and 2). Assuming optical depth in He II continuum below 1 one obtains from $N(\mathrm{He}$ II $)=\eta \cdot N(\mathrm{HI})<$ $5 \times 10^{17} \mathrm{~cm}^{-2}$ the vlue of $\eta \sim 10$ which assumes a low ionization parameter (high gas density) and a hard ionizing spectrum.

A hard ionizing continuum is also required by the presence of $\mathrm{N} \mathrm{V}$, otherwise we obtain $[\mathrm{N} / \mathrm{C}]>0$. Such overabundance of 


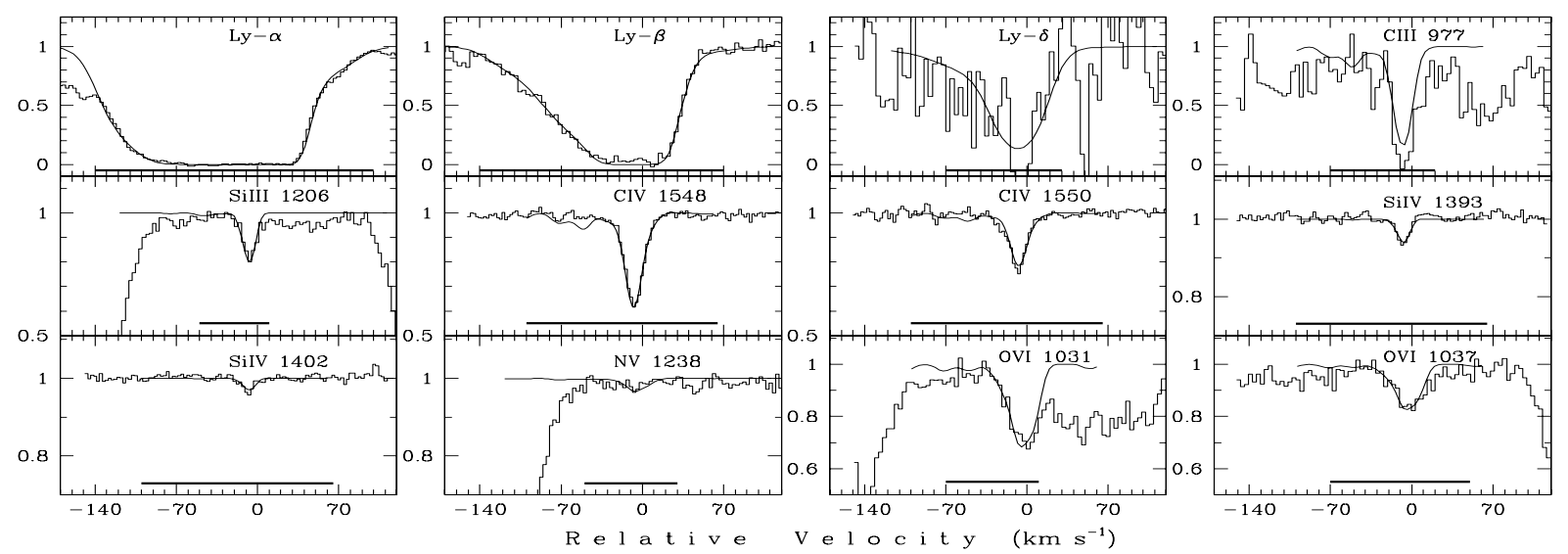

Fig. 16. Same as Fig. 3 but for the $z_{\text {abs }}=2.379$ system towards HS $1700+6416$. Synthetic profiles (smooth curves) were calculated with the recovered ionizing spectrum shown by the dotted line in Fig. 13. The zero radial velocity is fixed at $z=2.3799$. The estimated physical parameters are listed in Table 3, Col. 5.

nitrogen is predicted at high over solar metallicities by chemical evolution models (e.g. Hamann \& Ferland 1999), but at $Z \sim Z_{\odot}$ still deficit of nitrogen, $[\mathrm{N} / \mathrm{C}]<0$, is measured (e.g. Srianand \& Petitjean 2001; Reimers et al. 2005a; Jenkins et al. 2005). In principle, we cannot exclude a possibility that the present system is out of equilibrium and $\mathrm{N} \mathrm{V}$ is still overionized (i.e. not in the ionization equilibrium with the background radiation). The $\mathrm{O}$ VI lines could clear up the actual physical conditions but they fall beyond the observable range. However, we do not observe any velocity shifts between the $\mathrm{N} v$ lines and all other ionic transitions which could indicate a multiphase nature of the absorbing gas. This makes "normal" (equilibrium) conditions preferable.

Another very important clue to the physical properties of the absorbing gas deliver silicon ions: the observed ratios of $\mathrm{Si} \mathrm{IV/Si} \mathrm{III} \simeq 1$ and Si IV/Si II $\simeq 10$ can be realized only at metallicities near solar values. As already explained in Sect. 3.1.2, this stems from a strong dependence of silicon ion fractions on the temperature which at high metallicities overrides dependence on the spectral shape of the $U V B$.

Taking together, these considerations allow us to design a possible shape of the ionizing continuum. It is shown in Fig. 13 by the long-dashed line with the derived physical parameters given in Table 3, Col. 6 and synthetic profiles plotted by the solid lines in Fig. 17. The red wing of a sole hydrogen line is inconsistent with the assumption of constant metallicity inside the absorber and we calculated it using the density-velocity distribution obtained from metal lines. As mentioned above, the available silicon lines require metallicity close to solar. However, with $Z \simeq 0.5 Z_{\odot}$ we obtained $N(\mathrm{HI})=2.5 \times 10^{16} \mathrm{~cm}^{-2}$ which is already half that estimated from the Lyman edge in low resolution spectrum. A possible explanation of this discrepancy may be that we observe a high metallicity $\left(Z \sim Z_{\odot}\right)$ blob with $N(\mathrm{H} \mathrm{I}) \sim(1-2) \times 10^{16} \mathrm{~cm}^{-2}$ embedded in a cloud or a chain of clouds with a joint column density $N(\mathrm{HI}) \sim(3-4) \times 10^{16} \mathrm{~cm}^{-2}$. To confirm this assumption, higher order H I Lyman series lines are needed.

Because of this uncertainty of $N(\mathrm{HI})$ in the metal-bearing cloud and unknown element ratios, the spectral shape plotted in Fig. 13 is not unique. However, it delivers quite reasonable and self-consistent physical parameters and, thus, can be considered as a plausible solution. In any case, the spectral indices $\alpha>-1.2$ in the range $1<E<4$ Ryd are favored since for lower indices one would obtain $\mathrm{C} / \mathrm{Si}$ above solar which has never been observed and is not predicted theoretically.
Taking together, the high metallicity of the blob and hard ionizing spectrum allow to suggest the presence of a QSO/AGN close to the line of sight.

\section{Discussion}

The spectral shape of the background ionizing radiation in the energy range $1<E<10$ Ryd is reconstructed from opticallythin metal absorption-line systems observed in QSO spectra at $z \approx 1.80$ and $2.38<z<2.94$. The spectral shape of the $U V B$ does fluctuate, however these fluctuations are ruled by different sources at two considered redshift intervals.

In the range $2.38<z<2.94$, the spectral shape is mostly affected by radiative transfer processes in the clumpy IGM involving absorption in both the He II Ly- $\alpha$ and Lyman continuum. We confirm our result from Paper I: the $U V B$ at $2.4<$ $z_{\text {abs }}<3.0$ demonstrates the intensity depletion between 3 and 4 Ryd caused by the absorption of the He II Ly $\alpha$ photons in the IGM (He II Gunn-Petersen effect). None of the recovered $U V B$ shapes shows features which can be attributed to the contribution of radiation from soft sources like starburst galaxies. Such contribution would lead to a very steep slope at $E>1$ Ryd $(\alpha<-2.0$ at $1<E<3$ Ryd) and reduced intensity at 3 Ryd (see Figs. 1 and 3 in Giroux \& Shull 1997) whereas the metalline systems considered here require relatively hard $(\alpha>-1.6)$ spectral shapes with pronounced peaks at 3 Ryd combined with strong attenuation at $E>4$ Ryd - features unambiguously related to the absorption in the He II Lyman continuum and subsequent re-emission of recombinant He II Ly- $\alpha$.

From the depth of the GP trough in the recovered $U V B$ spectra we can estimate the effective $\operatorname{He}$ II Ly $\alpha$ opacity of the IGM, $\tau_{\mathrm{GP}}^{\mathrm{He}}$. Figure 18 shows the obtained redshift evolution of $\tau_{\mathrm{GP}}^{\mathrm{He}}$ (filled symbols) together with the available directly measured He II Ly $\alpha$ opacities (open symbols) along different sightlines. The concordance between the recovered values and the direct measurements is good although our estimations are systematically higher by $20-40 \%(\sim 1-2 \sigma)$. This difference can be explained by the following reasons. Firstly, the approximation of the continuum depression between 3 and 4 Ryd by a straight step does not take into account a winding shape of the actual GP trough. Secondly, the depth of this step is determined mostly from the analysis of carbon lines C II, C III, and C IV (see Sect. 2). However, the ionization potential of C III (3.52 Ryd) is close to the onset of the $\mathrm{He}$ II $\mathrm{Ly} \beta$ forest (3.56 Ryd) and an 


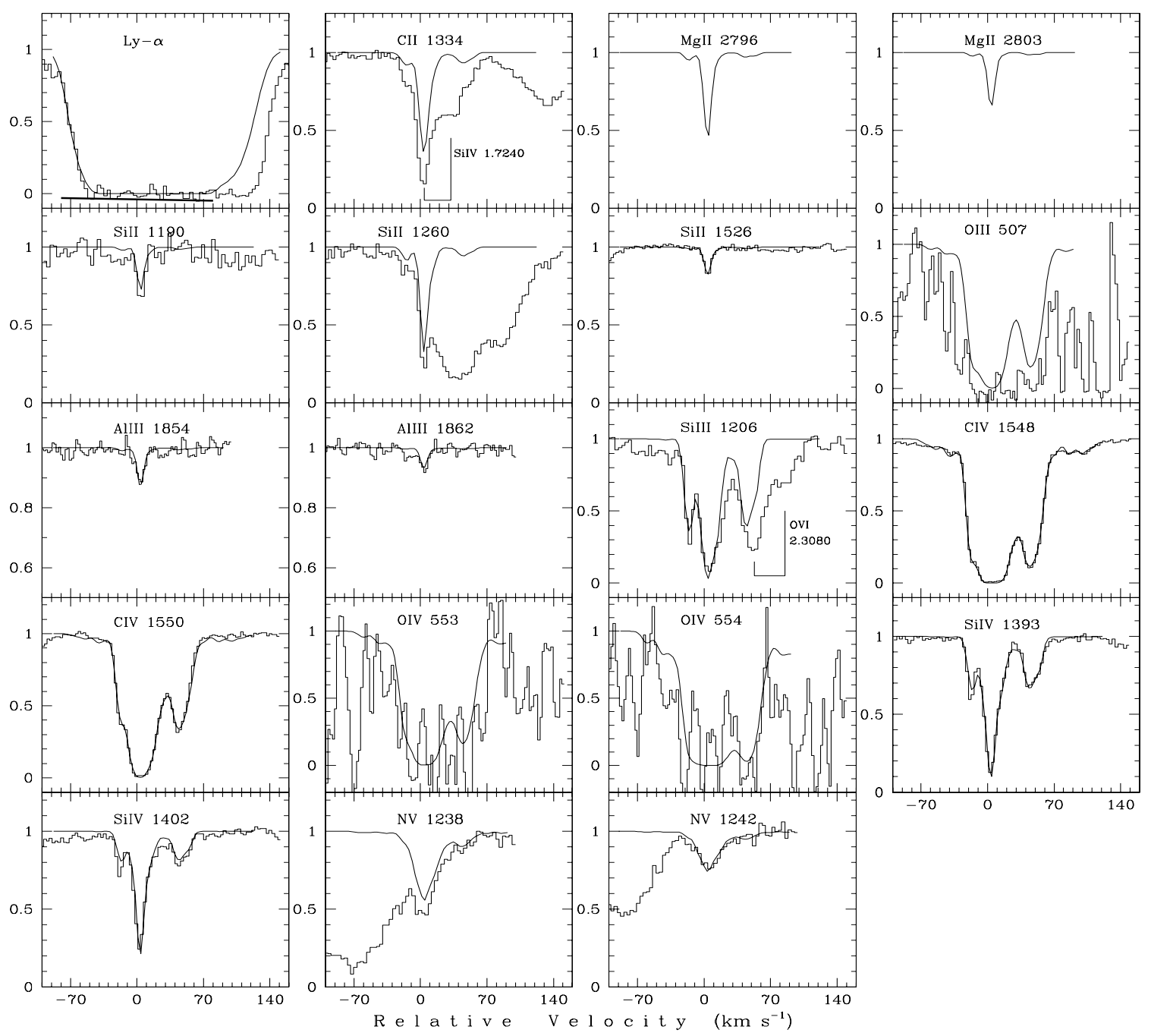

Fig. 17. Same as Fig. 3 but for the $z_{\text {abs }}=1.845$ system towards HS 1700+6416. Synthetic profiles (smooth curves) were calculated with the recovered ionizing spectrum shown by the long-dashed line in Fig. 13. The zero radial velocity is fixed at $z=1.8450$. The estimated physical parameters are listed in Table 3, Col. 6. Blends are indicated by tick marks.

additional GP absorption in He II Ly $\beta$ can affect the fraction of this ion and the subsequent C IV. These may lead to the overestimation of $\tau_{\mathrm{GP}}^{\mathrm{He} I \mathrm{I}}$ by $0.1-0.2$ in our method. On the other hand, estimations of $\tau_{\mathrm{GP}}^{\mathrm{He} I \mathrm{I}}$ from FUSE data may give too small values due to problems in background subtraction at short wavelengths corresponding to $z<2.55$ (see Sect. 2 in F06).

Analysis of the close absorption systems $\left(z_{\mathrm{abs}}=2.735\right.$ and 2.739 towards HE 2347-4342 and $z_{\text {abs }}=2.433$ and 2.438 towards HS 1700+6416) revealed two-three times fluctuations in $\eta$ between them. These systems share the same $U V B$, and the fluctuations in $\eta$ are caused entirely by the variations in the absorber gas density.

Now compare the $z_{\mathrm{abs}}=2.433$ and 1.845 systems. Both of them show similar $U$ and $n_{\mathrm{H}}$, but their $\eta$ values differ by about 3 times (Table 3 ). In this case we observe the effect of different spectral softness (see Fig. 13).

Figure 19 shows the dependence of $\eta_{\text {model }}=Z_{\mathrm{He}} \Upsilon_{\mathrm{HeII}} / \Upsilon_{\mathrm{HI}}$ on the ionization parameter $U$ calculated with CLOUDY for optically thin case assuming different ionizing spectra. In case of a constant gas density inside the absorber, $\eta_{\text {model }}=\eta$. When the gas density fluctuates within the absorber, $\eta$ is defined as a density weighted mean of the $\eta(U)$ values, thus being lower than $\eta_{\text {model }}$ corresponding to the mean ionization parameter $U_{0}$ (see Eqs. (24), (25) in LAK). It is seen that in the range $-3<$ $\log (U)<-1$ relevant for optically thin high redshift intergalactic absorbers the $\eta_{\text {model-curve demonstrates a large gradi- }}$ ent which becomes steeper with the softening of the $U V B$ at $E>4$ Ryd. Moreover, the interval of $\eta_{\text {model }}$ values corresponding to $-3<\log (U)<-1$ also expands. Thus, in general, the scatter in the measured values of $\eta$ can arise from the combined action of differences in the mean gas density between the absorbers and variable softness of the ionizing radiation due to absorption in the He II Lyman continuum. We can also predict that the scatter in $\eta$ should decrease with decreasing $z$ due to progressive hardening of the $U V B$ spectrum.

Figure 19 explains also the correlation between low H I column densities and high $\eta$ values: weak absorbers tend to have lower gas densities and thus are shifted to the right part of the $\eta(U)$ curve. This is also one of the reasons why $\eta$ in voids are systematically higher than in the filaments: by definition, voids consist of weak H I absorbers. Another reason is a possible shielding of the UV radiation in voids by the surrounding clouds which can soften the ionizing spectra. This is illustrated by the $z_{\text {abs }}=2.735,2.739$ and 2.741 absorbers towards HE 2347-4342 located at the boundary of the void at $2.718<z<2.727$ (Fig. 5 in Z04). These absorbers belong probably to the filamentary 


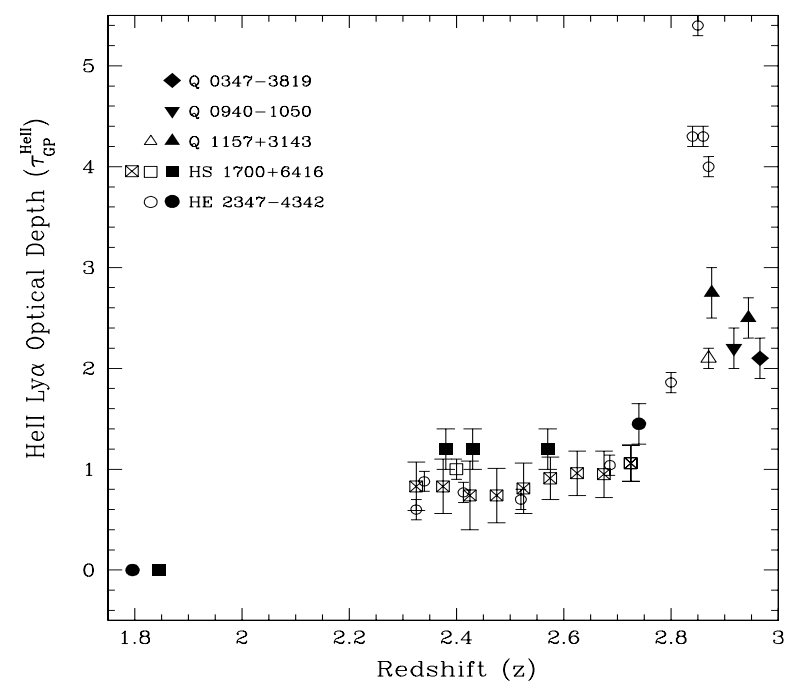

Fig. 18. Redshift dependence of the He II Ly $\alpha$ opacity, $\tau_{\mathrm{GP}}^{\mathrm{He}}(z)$. Open symbols show direct measurements from HE 2347-4342 (Zheng et al. 2004), HS 1700+6416 (open square - Davidsen et al. 1996, crossed open square - Fechner et al. 2006a), and Q 1157+3143 (Reimers et al. $2005 \mathrm{~b}$ ). Filled symbols represent the values of $\tau_{\mathrm{GP}}^{\mathrm{He} I \mathrm{I}}$ estimated from the restored spectral shapes of the $U V B$ ionizing radiation: diamond and reverse triangle - Paper I; triangles, squares and circles - the present paper. Error bars correspond to $1 \sigma$ confidence level.

structure and form a layer with a linear size of $\sim 300 \mathrm{kpc}^{3}$. Table 2 from Z04 shows that the $\eta$ values in the void at $2.718<$ $z<2.727$ are significantly higher than in the void at $2.816<$ $z<2.823$. The latter has no strong H I absorbers at its boundaries and lies close to the quasar HE 2347-4342, i.e. the $U V B$ in this void can be quite hard. However, over large ranges in $z$ the correlation "low $N(\mathrm{HI})-$ high $\eta$ " $\left[N(\mathrm{HI})=10^{12}-10^{13} \mathrm{~cm}^{-2}\right]$ should be weak due to superposition of $U V B$ s with variable softness: harder $U V B$ s give a significantly lower $\eta$ especially for low gas density (and, in general, low column density) absorbers, thus effectively lowering the left side of the " $N(\mathrm{HI})-\eta$ " distribution. As for stronger $\mathrm{H} \mathrm{I}$ absorbers $\left[N(\mathrm{HI})=10^{13}-10^{15} \mathrm{~cm}^{-2}\right]$, they have usually higher gas densities (lower $U$ values) and according to Fig. 19 their $\eta$ values are less sensitive to the variations of $U V B$. Thus, the correlation "high $N(\mathrm{HI})-$ low $\eta$ " should hold over all redshifts. This is in concordance with direct measurements of $\eta$ from the H I/He II Ly $\alpha$ forest in F06 (see their Fig. 10) and Fechner \& Reimers (2007).

As for $U V B$ s recovered at $z \simeq 1.8$, they are very hard at $E>3 \mathrm{Ryd}$ - much harder than predicted by the HM model (Fig. 20). The reason for this discrepancy was already given in our Paper II: HM used a biased statistics of the Ly $\alpha$ clouds at $z_{\text {abs }} \lesssim 1.8$ with too many strong Ly $\alpha$ absorbers leading to a significant underestimation of the $U V B$ intensity at $E>3$ Ryd. From the depth of the He II break at 4 Ryd we can calculate the effective He II column density which gives rise to this break (Table 4). It is seen that the values of $N(\mathrm{He}$ II) are spread by maximum $40 \%$ around the median $1.2 \times 10^{18} \mathrm{~cm}^{-2}$. Besides, the recovered ionizing spectra show a diversity of shapes with a tendency to high spectral indices at $1<E<4$ Ryd ( $\alpha>-1.4$, median $\alpha=-1.0$ ). These spectra are likely to be intrinsic spectra of quasars, i.e. not

${ }^{3}$ It is interesting to note that other absorbers from our study with characteristics similar to those of the complex at $z=2.735-2.741$, namely the $z_{\mathrm{abs}}=2.917$ system towards HE 0940-1080 (Sect. 3.1, Paper I) and the $z_{\text {abs }}=2.875$ system towards Q $1157+3143$ (Sect. 3.2.3, present paper), also lie at the boundary of a void.

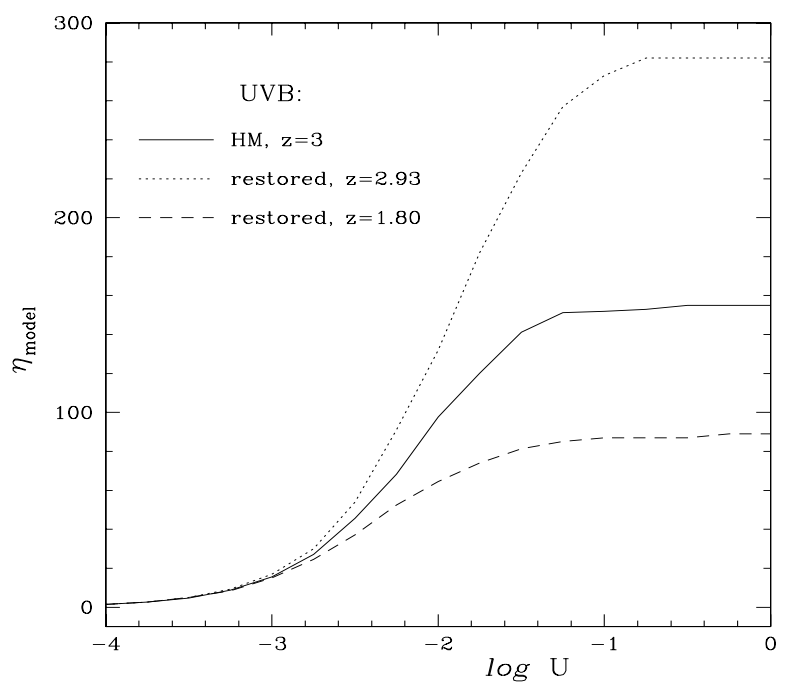

Fig. 19. Dependence of $\eta_{\text {model }}$ on the ionization parameter $U$ for different spectral shapes of the ionizing radiation: $\mathrm{HM}$ at $z=3$ (solid line), and $U V B$ restored at $z=2.93$ (dotted line) and $z=1.80$ (dashed line) which are, respectively, softer and harder than the HM spectrum. The $U V B$ at $z=2.93$ (Q 1157+3143) is shown in Fig. 9, and at $z=1.80$ (HS $0747+4259)-$ in Fig. 20. The adopted metallicity is $Z=0.01 Z_{\odot}$.

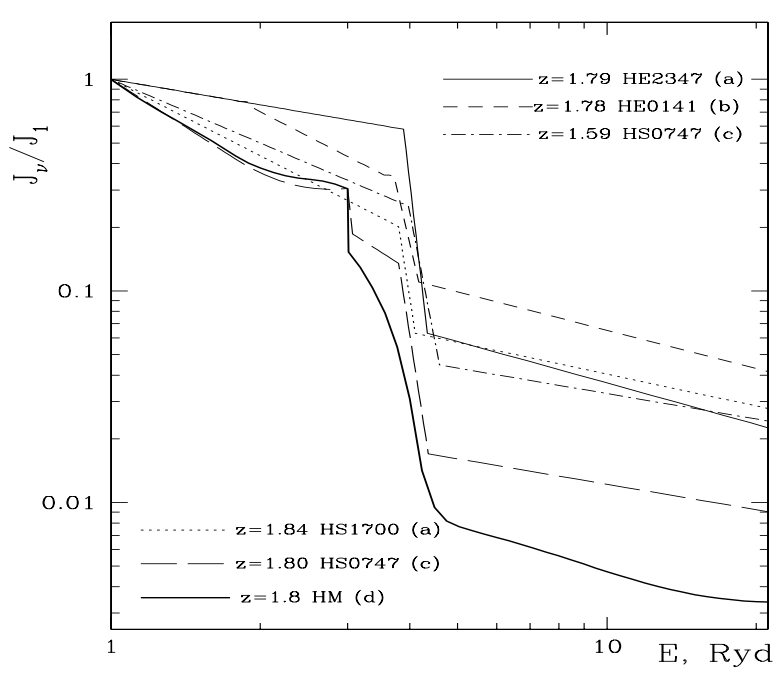

Fig. 20. The difference between the $U V B$ predicted by $\mathrm{HM}$ at $z=1.8$ (thick line) and those restored by the MCISS procedure (thin lines). The data are taken from (a) - the present paper, quasars HE 2347-4342 and HS 1700+6416, (b) - Reimers et al. (2005a), quasar HE 0141-3932, (c) - Paper II, quasar HS 0747+4259, and (d) - Haardt \& Madau (1996).

processed by the IGM. In order to cause a noticeable absorption in the He II Lyman continuum, the intervening absorbers should have $N($ He II $)>5 \times 10^{17} \mathrm{~cm}^{-2}$. According to Fig. 19, the corresponding lower limit on $N(\mathrm{H} \mathrm{I})$ will be $3.0 \times 10^{15} \mathrm{~cm}^{-2}$ for the HM spectrum at $z=3$ (middle curve) and $1.8 \times 10^{15} \mathrm{~cm}^{-2}$ and $5.5 \times 10^{15} \mathrm{~cm}^{-2}$ for the softer and harder spectra (upper and lower curves, respectively). From statistics of the Ly $\alpha$ forest (e.g., Kim et al. 2002) it is well known that strong H I absorbers with $N(\mathrm{H} \mathrm{I})>10^{15} \mathrm{~cm}^{-2}$ become more rare with decreasing redshift. This results in less absorption at the He II ionization edge leading to a harder $U V B$. To produce $N($ He II $)>5 \times 10^{17} \mathrm{~cm}^{-2}$, harder spectra, in turn, require more stronger $\mathrm{HI}$ absorbers which are even more rare. Thus, by $z \simeq 1.8$ a large part of the QSO/AGN emitted radiation remains unprocessed. 
Table 4. He II column densities estimated from the ionizing spectra shown in Fig. 20.

\begin{tabular}{cccc}
\hline \hline Quasar & $z_{\text {abs }}$ & $\begin{array}{c}\tau_{\mathrm{c}}^{\mathrm{He} \text { II }} \\
\text { (at 4 Ryd })\end{array}$ & $\begin{array}{c}N(\mathrm{He} \mathrm{II}) \\
\left(\mathrm{cm}^{-2}\right)\end{array}$ \\
\hline HE 0141-3932 & 1.78 & 1.2 & $0.7 \mathrm{E} 18$ \\
HS 0747+4259 & 1.80 & 2.0 & $1.3 \mathrm{E} 18$ \\
& 1.59 & 1.8 & $1.2 \mathrm{E} 18$ \\
HS 1700+6416 & 1.84 & 1.2 & $0.7 \mathrm{E} 18$ \\
HE 2347-4342 & 1.79 & 2.2 & $1.4 \mathrm{E} 18$ \\
\hline
\end{tabular}

In support to this consideration the following observationbased arguments can be given. Using a combination of several hard X-ray surveys, Ueda et al. (2003) investigated cosmological evolution of the AGN luminosity function. They found that the comoving spatial density of most luminous AGNs (quasars) peaks at $z \simeq 2$ and after that decreases rapidly, but spatial density of less luminous but orders of magnitude more numerous AGNs continues to rise till $z \lessgtr 1$. On the other hand, a FUSE survey of the AGNs $(z<1)$ by Scott et al. (2004) shows that lower luminosity AGNs tend to have harder spectral slopes (median $\alpha=-0.6)$ in the range $650-1000 \AA$ (0.9-1.4 Ryd).

Thus, we can conclude that a significant hardening of the $U V B$ spectrum occurs between $z=2.38$ and $z=1.80$. According to Fig. 19, this hardening would result in decrease of $\eta$. The redshift evolution of $\eta$ reported in Fechner \& Reimers (2007) allows to suggest that hardening starts even earlier, somewhere at $z \sim 2.5$. At the moment we cannot trace the $U V B$ evolution between $z=2.5$ and $z=1.80$ because of scarcity of our absorbers in this redshift interval. This will be a topic of our future studies.

\section{Conclusions}

1. Using the MCISS method based on realistic models of line formation in stochastic media and adequate computational technique, we restored the spectral shapes of the underlying ionizing radiation from optically thin metal-line systems. The $U V B$ spectral shape does fluctuate in the redshift range studied, $1.8<z<2.94$. However, the fluctuations at $z \lesssim 1.8$ and $2.4<z<2.94$ are ruled by different physical reasons. At $2.4<z<2.94$, the fluctuations arise due to radiative transfer processes in the clumpy IGM, whereas at $z \lesssim 1.8$ the IGM becomes almost transparent both in the $\mathrm{H}$ I and He II Lyman continua and the variability of spectral shapes comes from diversity of spectral indices of the intrinsic QSO/AGN spectra.

2. At $2.4<z<2.94$, the recovered spectral shapes show the intensity depression between 3 and 4 Ryd which is interpreted as a manifestation of the He II Gunn-Peterson effect. The values of the mean He II Ly $\alpha$ opacity calculated from the depth of the recovered GP trough correspond well (within 1-2 $\sigma$ ) to $\tau_{\mathrm{GP}}^{\mathrm{He}}$ II directly measured from the H I/He II Ly $\alpha$ forest towards the quasars studied. This concordance confirms the applicability of the MCISS procedure for studying He II absorption in the IGM.

3. The scatter of $\eta=N(\mathrm{He} \mathrm{II}) / N(\mathrm{HI})$ and anti-correlation between $N(\mathrm{HI})$ and $\eta$ can be explained by the combined action of the spectral shape variability due to radiative transfer in the clumpy IGM and differences in the mean gas densities in the absorbing clouds. Progressive hardening of the recovered $U V B$ towards lower $z$ explains the corresponding decrease of the median $\eta$-values reported in F06 and Z04.
4. All recovered $U V B \mathrm{~s}$ are produced by QSO/AGN radiation processed by the clumpy IGM and do not show features which can be attributed to the input of radiation from soft sources like starburst galaxies. The metal systems considered in the present paper and in Paper I have overdensities 10-100 implying that radiative transfer effects both in the cloud and in the IGM can be significant. This means that for regions with smaller overdensities the $U V B$ should be harder in general. However, such regions (voids) can be bounded by filament structures optically thin in H I but thick in He II. In this case, the incident radiation is effectively shielded and the $U V B$ in voids becomes softer.

Acknowledgements. The work of IDA and SAL is partly supported by the RFBR grant No. 06-02-16489 and by the Federal Agency for Science and Innovations (grant NSh 9879.2006.2). CF is supported by the DFG under RE 353/49-1. DT is supported in part by NSF grant AST 0507717 and NASA grants NAG5-13113 and HST-AR-10688.01-A.

\section{References}

Agafonova, I. I., Centurión, M., Levshakov, S. A., \& Molaro, P. 2005, A\&A, 441, 9 [Paper I]

Asplund, M., Grevesse, N., \& Sauval, A. J. 2004, Nucl. Phys. A, 777, 1

Bolton, J. S., Haehnelt, M. G., Viel, M., \& Carswell, R. F. 2006, MNRAS, 366, 1378

Cayrel, R., Depagne, E., Spite, M., et al. 2004, A\&A, 416, 1117

Davidsen, A. F., Kriss, G. A., \& Zheng, W. 1996, Nature, 380, 47

Erni, P., Richter, P., Ledoux, C., \& Petitjean, P. 2006, A\&A, 451, 19

Fardal, M. A., Giroux, M. L., \& Shull, M. 1998, AJ, 115, 2206

Fechner, C., \& Reimers, D. 2007, A\&A, 461, 847

Fechner, C., Reimers, D., Kriss, G. A., et al. 2006a, A\&A, 455, 91 (F06)

Fechner, C., Reimers, D., Songaila, A., et al. 2006b, A\&A, 455, 73

Ferland, G. J. 1997, A Brief Introduction to Cloudy (Internal Rep., Lexington: Univ. Kentucky)

Ganguly, R., Charlton, J. C., \& Bond, N. A. 2001, ApJ, 553, L101

Giroux, M. L., \& Shull, J. M. 1997, AJ, 113, 1505

Haardt, F., \& Madau, P. 1996, ApJ, 461, 20 (HM)

Hamann, F., \& Ferland, G. 1999, ARA\&A, 37, 487

Holweger, H. 2001, in Solar and Galactic Composition, ed. R. F. Wimmer-Schweingruber, AIP Conf. Proc., 598, 23

Jenkins, E. B., Bowen, D. V., Tripp, T. M., \& Sembach, K. R. 2005, ApJ, 623, 767

Kim, T.-S., Carswell, R. F., Cristiani, S., D’Odorico, S., \& Giallongo, E. 2002, MNRAS, 335, 555

Kirkman, D., \& Tytler, D. 1999, ApJ, 512, L5

Levshakov, S. A., Agafonova, I. I., \& Kegel, W. H. 2000, A\&A, 360, 833 (LAK)

Levshakov, S. A., Agafonova, I. I., Centurión, M., \& Mazets, I. E. 2002, A\&A, 383,813

Levshakov, S. A., Agafonova, I. I., Reimers, D., \& Baade, R. 2003a, A\&A, 404, 449

Levshakov, S. A., Agafonova, I. I., D’Odorico, S., Wolfe, A. M., \& Dessauges-Zavadsky, M. 2003b, ApJ, 582, 596

Maselli, A., \& Ferrara, A. 2005, MNRAS, 364, 1429

Morton, D. C. 2003, ApJS, 149, 205

Reimers, D., Vogel, S., Hagen, H.-J., et al. 1992, Nature, 360, 561

Reimers, D., Janknecht, E., Fechner, C., et al. 2005a, A\&A, 435, 17

Reimers, D., Fechner, C., Hagen, H.-J., et al. 2005b, A\&A, 442, 63

Reimers, D., Agafonova, I. I., Levshakov, S. A., et al. 2006, A\&A, 449, 9 (Paper II)

Scott, J. E., Kriss, G. A., Brotherton, M., et al. 2004, ApJ, 615, 135

Shull, J. M., Tumlinson, J., Giroux, M. L., Kriss, G. A., \& Reimers, D. 2004, ApJ, 600, 570

Simcoe, R. A., Sargent, W. L. W., \& Rauch, M. 2002, ApJ, 578, 737

Simcoe, R. A., Sargent, W. L. W., Rauch, M., \& Becker, G. 2006, ApJ, 637, 648

Srianand, R., \& Petitjean, P. 2001, A\&A, 373, 816

Ueda, M., Akiyama, M., Ohta, K., \& Miyaji, T. 2003, ApJ, 598, 886

Verner, D. A., Barthel, P. D., \& Tytler, D. 1994, A\&AS, 108, 287

Vogel, S., \& Reimers, D. 1995, A\&A, 294, 377

Zheng, W., Kriss, G. A., Deharveng, J.-M., et al. 2004, ApJ, 605, 631 (Z04) 